\title{
Reduced basis methods for nonlocal diffusion problems with random input data
}

\author{
Qingguang Guan ${ }^{\mathrm{a}}$, Max Gunzburger ${ }^{\mathrm{a}}$, Clayton G. Webster ${ }^{\mathrm{b}, \mathrm{c}}$, Guannan Zhang ${ }^{\mathrm{b}}$ \\ ${ }^{a}$ Department of Scientific Computing, Florida State University, Tallahassee, FL 32306 \\ ${ }^{b}$ Computational and Applied Mathematics, Oak Ridge National Laboratory, Oak Ridge, TN 37831 \\ ${ }^{c}$ Department of Mathematics, University of Tennessee, Knoxville, TN 37996
}

\begin{abstract}
The construction, analysis, and application of reduced-basis methods for uncertainty quantification problems involving nonlocal diffusion problems with random input data is the subject of this work. Because of the lack of sparsity of discretized nonlocal models relative to analogous local partial differential equation models, the need for reducedorder modeling is much more acute in the nonlocal setting. In this effort, we develop reduced-basis approximations for nonlocal diffusion equations with affine random coefficients. Efficiency estimates of the proposed greedy reduced-basis methods is provided. Numerical examples are used to illustrate the effect varying various model parameters have on the efficiency and accuracy of the reduced-basis method relative to the sparse grid interpolation using the full finite element method. It is shown that the proposed reduced-basis approach can indeed provide substantial savings over the standard sparse grid method when combined with full finite element solvers.
\end{abstract}

Keywords: reduced-basis methods, nonlocal diffusion, finite elements, uncertainty quantification

\section{Introduction}

In classical local models, interactions only occur due to contact and are described by partial differential equations (PDEs). However, in many settings (e.g., continuum mechanics [1, 2], image processing [3-6], fractional dynamics and diffusion [7], machine learning [8], and graph theory [9]), interactions are intrinsically nonlocal. Here, "nonlocal"

\footnotetext{
« This material is based upon work supported in part by the U.S. Air Force of Scientific Research under grants 1854-V521-12 and FA9550-15-1-0001; the U.S. Defense Advanced Research Projects Agency, Defense Sciences Office under contract and award HR0011619523 and 1868-A017-15; the U.S. Department of Energy, Office of Science, Office of Advanced Scientific Computing Research, Applied Mathematics program under contracts and awards ERKJ259, ERKJE45, DE-SC0009324, and DE-SC0010678; and by the Laboratory Directed Research and Development program at the Oak Ridge National Laboratory, which is operated by UT-Battelle, LLC., for the U.S. Department of Energy under contract DE-AC0500OR22725.
} 
is used to describe long-range spatial interactions. For example, in solid mechanics, the strain energy density at a point $\mathbf{x}$ can depend on points in a neighborhood of $\mathbf{x}$ having non-zero volume. This is in contrast to local PDE models where interactions occur only in an infinitesimal neighborhood of the point $\mathbf{x}$. Further, without some interventions, PDEs cannot model discontinuous behavior such as crack nucleation and propagation. Nonlocal models that are free of spatial derivatives can gracefully handle continuous and discontinuous behaviors such as fracture $[1,2]$. In image denosing, it is often reasonable to assume that the noise at any point is related directly to that any other point in the image, or at least to points in relevant regions, rather than just its immediate neighbors. This leads to the study of nonlocal operators in image analyses [3-6] that, similar to the nonlocal model in continuum mechanics, can process both structures (continuous) and textures (discontinuous) within the same framework. In the case considered here, i.e., diffusion, nonlocal interaction give rise to anomalous diffusion, i.e., to diffusive behavior that does not follow Fickian laws or that can be modeled by Brownian motions. In [10], a finite element framework and a thorough variational analysis is provided for nonlocal problems based on the nonlocal vector calculus recently developed in [11].

On the other hand, the inputs to a model, be they local or nonlocal, are often subject to uncertainties. For example, in the PDE setting, coefficients, forcing terms, and data in initial and boundary conditions may be uncertain. Thus, uncertainty quantification, i.e., the analysis and quantification of the uncertainties in the outputs of a model due to uncertainties in its inputs, has been of growing interest in recent years. For local problems modeled by differential equations with random input data, the classical Monte Carlo method is widely used. The appealing properties of this method are its ease of implementation based on existing deterministic codes and the independence of its convergence rate with respect to the number of input random variables. However, the $\mathcal{O}(1 / \sqrt{M})$ convergence rate of Monte Carlo methods is slow, where here $M$ denotes the number of sample points. Although this behavior can be somewhat alleviated by, e.g., Latin hypercube sampling [12, 13], quasi-Monte Carlo methods [14, 15], and multi-level Monte Carlo methods [16], convergence behavior is still too slow, given that in most settings, one has to solve the differential equation for every sample set of random parameters.

Several other methods exhibits a faster convergence rates, provided that the solution of the PDE is sufficiently smooth with respect to the random variables. For stochastic Galerkin methods (SGMs), the discretization with respect to the random parameters is effected using a Galerkin method, usually using global orthogonal polynomial approximations (referred to as polynomial chaos expansions) [17-21]. Stochastic collocation methods (SCMs) are another approach that achieves a fast convergence rate. SCMs falls within the class of sampling methods so that, like Monte-Carlo methods, only solutions at sample points in parameter space are needed in order to, e.g., evaluate a quantity of interest. A major challenge with SCMs is the selection of interpolation/quadrature points in parameter space. Tensor products of one-dimensional quadrature involve explosive growth in the number of quadrature points as the number of random parameters increases. Sparse-grid collocation methods [22-24, 33] are superior for reducing the number of sample points needed to achieve the same accuracy as do tensor product points. 
The efforts mentioned above attempt to reduce the costs of uncertainty quantification in the PDE setting by reducing the number of sample points in parameter space (i.e, reducing the number of times the PDE has to be solved) needed in order to achieve a desired accuracy. Other efforts are devoted to reducing the costs of obtaining an approximation of the solution of the PDE. To this end, reduced-basis methods were developed for parametrized PDEs [27-31] that can significantly reduce the solving cost.

Despite the recent interest in numerical methods for nonlocal problems, studies of such problems in the uncertainty quantification setting are not widely available in the literature. In this paper, we exploit the framework of $[10,11]$ to set nonlocal problems with random input data into a proper approximation space and develop reduced-basis methods for constructing surrogates of the solution of the nonlocal diffusion problem and computing statistical quantities of interest. It is important to note that the need for reduced-order models in settings that require multiple solutions of the model equations (such as uncertainty quantification or parameter optimization) is to a significantly greater extent for nonlocal models than it is for local PDE models. This is because, of the lack of sparsity, the cost of doing simulations in the former setting are usually much greater that it is in the latter setting.

The outline of this paper is as follows. In the remainder of this section, we review, in the deterministic setting, the nonlocal problems we consider. In $\S 2$, the stochastic nonlocal diffusion equation that is the focus of this paper is studied. In $\S 3$, greedy reduced-basis methods are considered and convergence results and computational costs are discussed. Then, in $\S 4$, a simplified reduced-basis method is introduced. In $\S 5$, numerical results are provided that illustrate the error estimates and computational costs of the both reducedbasis methods. Finally, in the Appendix, we provide a brief review of sparse-grids and sparse-grid interpolation, which is are ingredients in all the approaches discussed in this paper.

\subsection{The deterministic nonlocal problem}

The nonlocal problem we consider is the counterpart of the (local) partial differential equation problem

$$
\begin{aligned}
-\nabla \cdot(\kappa(\mathbf{x}) \nabla u(\mathbf{x})) & =f(\mathbf{x}) & & \text { for } \mathbf{x} \in D, \\
u(\mathbf{x}) & =0 & & \text { for } \mathbf{x} \in \partial D,
\end{aligned}
$$

where $D \subset \mathbb{R}^{d}$ denotes an open bounded Lipschitz spatial domain having boundary $\partial D$ and where $d=1,2$, or 3 denotes the spatial dimension.

Let $\gamma\left(\mathbf{x}, \mathbf{x}^{\prime}\right): \mathbb{R}^{d} \times \mathbb{R}^{d} \rightarrow \mathbb{R}$ denote a non-negative function such that $\gamma\left(\mathbf{x}, \mathbf{x}^{\prime}\right)=\gamma\left(\mathbf{x}^{\prime}, \mathbf{x}\right)$ for all $\mathbf{x}, \mathbf{x}^{\prime} \in \mathbb{R}^{d}$. Define the interaction domain $D_{I} \subset \mathbb{R}^{d} \backslash D$ corresponding to $D$ by

$$
D_{I}=\left\{\mathbf{x}^{\prime} \in \mathbb{R}^{d} \backslash D: \gamma\left(\mathbf{x}, \mathbf{x}^{\prime}\right) \neq \mathbf{0} \forall \mathbf{x} \in D\right\} .
$$

$D_{I}$ consists of the points outside of $D$ that interact with the points in $D$; in this sense, $D_{I}$, which in general has finite volume, plays the role that $\partial D$ has for local problems. 
The nonlocal problem we consider has the form

$$
\left\{\begin{aligned}
\int_{D \cup D_{I}}\left(u(\mathbf{x})-u\left(\mathbf{x}^{\prime}\right)\right) \theta\left(\mathbf{x}, \mathbf{x}^{\prime}\right) \gamma\left(\mathbf{x}, \mathbf{x}^{\prime}\right) d \mathbf{x}^{\prime}=f(\mathbf{x}) & & \text { for } \mathbf{x} \in D \\
u(\mathbf{x})=0 & & \text { for } \mathbf{x} \in D_{I}
\end{aligned}\right.
$$

where $\theta\left(\mathbf{x}, \mathbf{x}^{\prime}\right): \mathbb{R}^{d} \times \mathbb{R}^{d} \rightarrow \mathbb{R}$ also denotes a non-negative function such that $\theta\left(\mathbf{x}, \mathbf{x}^{\prime}\right)=$ $\theta\left(\mathbf{x}^{\prime}, \mathbf{x}\right)$ for all $\mathbf{x}, \mathbf{x}^{\prime} \in \mathbb{R}^{d}$. Note that we keep $\theta\left(\mathbf{x}, \mathbf{x}^{\prime}\right)$ and $\gamma\left(\mathbf{x}, \mathbf{x}^{\prime}\right)$ separate because $\gamma$ is a kernel function that determines the qualitative nature, e.g., the regularity, of the solution $u(\mathbf{x})$, whereas $\theta$ is a constitutive function, e.g., it plays the role of a diffusivity coefficient; a common choice is $\theta\left(\mathbf{x}^{\prime}, \mathbf{x}\right)=\frac{1}{2}\left(\kappa(\mathbf{x})+\kappa\left(\mathbf{x}^{\prime}\right)\right)$.

The first equation in (2) mimics the differential equation in (1). However, because of nonlocality, the Dirichlet boundary condition in (1) is replaced by a Dirichlet volume constraint, i.e., by the second equation in (2), where by "volume constraint" we mean a constraint that acts on a set $D_{I} \subset \mathbb{R}^{d}$ having positive measure, as opposed to the boundary condition in (1) which acts on a set $\partial D \subset \mathbb{R}^{d}$ of measure zero.

Specific nonlocal problems are defined by the selection of the kernel $\gamma\left(\mathbf{x}, \mathbf{x}^{\prime}\right)$ in $(2)$. Many such kernels have been considered in the literatures (see, e.g., [10, 25, 26]) for all of which the well-posedness of the nonlocal problem has been proved. Here we only consider the kernel

$$
\gamma\left(\mathbf{x}, \mathbf{x}^{\prime}\right)= \begin{cases}0 & \text { if }\left|\mathbf{x}-\mathbf{x}^{\prime}\right|>\delta \\ \frac{1}{\left|\mathbf{x}^{\prime}-\mathbf{x}\right|^{d+2 s}} & \text { if }\left|\mathbf{x}-\mathbf{x}^{\prime}\right| \leq \delta\end{cases}
$$

for $s \in[0,1)$. The analytical and computational results given in this paper can be extended to the other kernels considered in, e.g., [10, 25, 26]. For $s \in(0,1), \theta=1, \delta=\infty$, and $\gamma$ given by (3), (2) is equivalent to the fractional Laplacian problem $(-\Delta)^{s} u=f$ posed on a bounded domain $D \subset \mathbb{R}^{d}$; see [10] for additional details.

To conduct variational analysis of the problem in (1), we define the bilinear form

$$
a(u, v):=\frac{1}{2} \int_{D \cup D_{I}} \int_{D \cup D_{I}}\left(u(\mathbf{x})-u\left(\mathbf{x}^{\prime}\right)\right)\left(v(\mathbf{x})-v\left(\mathbf{x}^{\prime}\right)\right) \theta\left(\mathbf{x}, \mathbf{x}^{\prime}\right) \gamma\left(\mathbf{x}, \mathbf{x}^{\prime}\right) d \mathbf{x}^{\prime} d \mathbf{x}
$$

the associated energy space

$$
\mathcal{V}\left(D \cup D_{I}\right):=\left\{v \in L^{2}\left(D \cup D_{I}\right): a(v, v)<\infty\right\}
$$

and the constrained energy space

$$
\mathcal{V}_{c}\left(D \cup D_{I}\right):=\left\{v \in \mathcal{V}\left(D \cup D_{I}\right): v=0 \text { a.e. on } D_{I}\right\}
$$

$\mathcal{V}\left(D \cup D_{I}\right)$ is a Hilbert space equipped with the inner product $(u, v)_{\mathcal{V}}:=a(u, v)+(u, v)$ and norm $\|u\|_{\mathcal{V}}:=(a(u, u)+(u, u))^{1 / 2}$, where $(\cdot, \cdot)=\int_{D \cup D_{I}} u v d \mathbf{x} ; \mathcal{V}_{c}\left(D \cup D_{I}\right)$ is also a Hilbert space with inner product $(u, v)_{\mathcal{V}_{c}}:=a(u, v)$ and norm $\|u\|_{\mathcal{V}_{c}}:=(a(u, u))^{1 / 2}$. From [10], we have 
Lemma 1. $\|u\|_{L^{2}\left(D \cup D_{I}\right)} \leq C_{P}\|u\|_{\mathcal{V}_{c}}$.

We also define the dual space $\mathcal{V}_{c}^{\prime}\left(D \cup D_{I}\right)$ corresponding to $\mathcal{V}_{c}\left(D \cup D_{I}\right)$ and, for any $f \in \mathcal{V}_{c}^{\prime}\left(D \cup D_{I}\right)$, the dual norm

$$
\|f\|_{\mathcal{V}_{c}^{\prime}}:=\sup _{v \in \mathcal{V}_{c}\left(D \cup D_{I}\right), v \neq 0} \frac{\int_{D \cup D_{I}} f v d \mathbf{x}}{\|v\|_{\mathcal{V}_{c}}} .
$$

A weak formulation corresponding to the nonlocal problem (2) is given by [10]: given $f \in \mathcal{V}_{c}^{\prime}\left(D \cup D_{I}\right)$, seek $u \in \mathcal{V}_{c}\left(D \cup D_{I}\right)$ such that

$$
a(u, v)=(f, v) \quad \forall v \in \mathcal{V}_{c}\left(D \cup D_{I}\right) .
$$

Because $a(u, v)$ defines an inner product on $\mathcal{V}_{c}\left(D \cup D_{I}\right)$, it is obvious by the Lax-Milgram theorem that the problem (5) is well posed, i.e., a unique solution exists and that solution satisfies the estimate

$$
\|u\|_{\mathcal{V}_{c}\left(D \cup D_{I}\right)} \leq\|f\|_{\mathcal{V}_{c}^{\prime}\left(D \cup D_{I}\right)} .
$$

For the kernel $\gamma\left(\mathbf{x}, \mathbf{x}^{\prime}\right)$ defined in (3) with $s \in(0,1)$, the space $\mathcal{V}\left(D \cup D_{I}\right)$ is the fractional Sobolev space $H^{s}\left(D \cup D_{I}\right)$ and the space $\mathcal{V}_{c}\left(D \cup D_{I}\right)$ is the space

$$
H_{c}^{s}\left(D \cup D_{I}\right):=\left\{v \in H^{s}\left(D \cup D_{I}\right): v=0 \text { on } D_{I}\right\} .
$$

Thus, for that kernel, the problem (2) has unique weak solution belonging to $H_{c}^{s}\left(D \cup D_{I}\right)$ and that solution satisfies the estimate

$$
\|u\|_{H^{s}\left(D \cup D_{I}\right)} \leq C\|f\|_{\left(H^{s}\left(D \cup D_{I}\right)\right)^{\prime}},
$$

where $C>0$ is independent of $u$ and $f$ and $\left(H^{s}\left(D \cup D_{I}\right)\right)^{\prime}$ denotes the dual space to $H_{c}^{s}\left(D \cup D_{I}\right)$. For $s=0$ and for the kernel $\gamma\left(\mathbf{x}, \mathbf{x}^{\prime}\right)$ defined in (3), there is no known characterization of the space $\mathcal{V}\left(D \cup D_{I}\right)$ in terms of known function spaces; what is known is that $\mathcal{V}\left(D \cup D_{I}\right)$ is a strict subspace of $L^{2}\left(D \cup D_{I}\right)$. Note that if the kernel $\gamma\left(\mathbf{x}, \mathbf{x}^{\prime}\right)$ is integrable, e.g., if $s<0$ in (3), then $\mathcal{V}\left(D \cup D_{I}\right)=L^{2}\left(D \cup D_{I}\right)$.

Continuous piecewise linear finite element methods have been applied to determine approximate solutions of the nonlocal diffusion problem (5); convergence results, which can be found in [10], are given in the following.

Proposition 1. Suppose $u \in H_{c}^{1+t}\left(D \cup D_{I}\right)$, where $u$ denotes the solution of (5) and where $0<s \leq t \leq 1$. Then, there exists a constant $C$, independent of the grid size $h$, such that, for sufficiently small $h$,

$$
\left\|u-u_{h}\right\|_{H^{s}\left(D \cup D_{I}\right)} \leq C h^{1+t-s}\|u\|_{H^{1+t}\left(D \cup D_{I}\right)},
$$

where $u_{h}$ denotes piecewise linear finite element approximation of $u$. 


\section{Nonlocal diffusion problems with random input data}

Throughout this paper, we assume that the noise is finite dimensional, i.e., the random inputs depend on a finite number $N$ of random variables, either because they have been approximated by, e.g., a truncated Karhunen-Loève expansion, or because the input itself is explicitly defined in terms of a finite number of random variables. In the latter case, we have a vector or random parameters $\boldsymbol{y} \in \Gamma \subset \mathbb{R}^{N}$ with a joint probability density function (PDF) $\rho(\boldsymbol{y}): \Gamma \rightarrow \mathbb{R}^{+}$. We assume throughout that the random parameters are independent, in which case $\Gamma=\prod_{n=1}^{N} \Gamma_{n}$ and $\rho(\boldsymbol{y})=\prod_{n=1}^{N} \rho_{n}\left(y_{n}\right)$; the one-dimensional PDFs $\rho_{n}\left(y_{n}\right): \Gamma_{n} \rightarrow \mathbb{R}$ corresponding the parameter $y_{n}, n=1, \ldots, N$, could all be different. In the case of parameterized random fields, the random variables $y_{n}, n=$ $1, \ldots, N$, are all drawn from the same one-dimensional PDF. For the sake of simplicity of exposition, we also assume that $\Gamma_{n}$ is bounded for all $n=1, \ldots, N$.

We now consider the case in which the coefficient $\theta$ and right-hand side $f$ in (2) are random, specifically, $\theta\left(\mathbf{x}, \mathbf{x}^{\prime}, \boldsymbol{y}\right): D \cup D_{I} \times D \cup D_{I} \times \Gamma \rightarrow \mathbb{R}$ and $f(\mathbf{x}, \boldsymbol{y}): D \times \Gamma \rightarrow \mathbb{R}$. We then seek, for any $\boldsymbol{y} \in \Gamma, u(\mathbf{x}, \boldsymbol{y})$ such that

$$
\left\{\begin{array}{rlrl}
\int_{D \cup D_{I}}\left(u(\mathbf{x}, \boldsymbol{y})-u\left(\mathbf{x}^{\prime}, \boldsymbol{y}\right)\right) & \theta\left(\mathbf{x}, \mathbf{x}^{\prime}, \boldsymbol{y}\right) \gamma\left(\mathbf{x}, \mathbf{x}^{\prime}\right) d \mathbf{x}^{\prime} & \\
& =f(\mathbf{x}, \boldsymbol{y}) & & \text { for } \mathbf{x} \in D, \boldsymbol{y} \in \Gamma \\
u(\mathbf{x}, \boldsymbol{y}) & =0 & & \text { for } \mathbf{x} \in D_{I}, \boldsymbol{y} \in \Gamma .
\end{array}\right.
$$

We assume that there exists $0<\theta_{\min } \leq \theta_{\max }<\infty$ such that

$$
\theta_{\min } \leq \theta\left(\mathbf{x}, \mathbf{x}^{\prime}, \boldsymbol{y}\right) \leq \theta_{\max } \quad \forall \mathbf{x}, \mathbf{x}^{\prime} \in D \cup D_{I} \quad \text { a.s. } \forall \boldsymbol{y} \in \Gamma .
$$

We also assume that $f$ has finite second moments, ${ }^{1}$ i.e., $\mathbb{E}\left[\|f\|_{L^{2}\left(D \cup D_{I}\right)}^{2}\right]<\infty$, that a.s. $f(\cdot, \boldsymbol{y}) \in L^{2}\left(D \cup D_{I}\right)$, and that a.s. $\theta(\cdot, \cdot, \cdot) \in L^{\infty}\left(D \cup D_{I}\right) \times L^{\infty}\left(D \cup D_{I}\right) \times L^{\infty}(\Gamma)$.

We define the Hilbert space $V_{\Gamma, \theta}$ as

$$
V_{\Gamma, \theta}:=\left\{\forall \boldsymbol{y} \in \Gamma, v(\mathbf{x}, \boldsymbol{y}) \in \mathcal{V}_{c}\left(D \cup D_{I}\right): \mathbb{E}\left[\|v\|_{\mathcal{V}_{c}}^{2}\right]<\infty\right\}
$$

with the norm $\|v\|_{\Gamma, \theta}=\sqrt{\mathbb{E}\left[\|v\|_{\mathcal{V}_{c}}^{2}\right]}$. Then, we have the following weak formulation corresponding to (8): find $u \in V_{\Gamma, \theta}$, such that

$$
\mathbb{E}[a(u, v)]=\int_{D} \mathbb{E}[f v] d \mathbf{x}, \quad \text { for all } v \in V_{\Gamma, \theta}
$$

As for the deterministic case, the well posedness of (10) follows easily from Lemma 1 and the Lax-Milgram theorem.

\footnotetext{
${ }^{1} \mathbb{E}[\cdot]$ denotes the mathematical expectation, i.e., $\mathbb{E}(v)=\int_{\Gamma} v(\boldsymbol{y}) \rho(\boldsymbol{y}) d \boldsymbol{y}$.
} 


\subsection{Discretization of the nonlocal problem}

We assume an affine dependence of the $\theta\left(\mathbf{x}, \mathbf{x}^{\prime} ; \boldsymbol{y}\right)$ on the parameters $\boldsymbol{y}$, i.e., we have

$$
\theta\left(\mathbf{x}, \mathbf{x}^{\prime} ; \boldsymbol{y}\right)=\theta_{0}\left(\mathbf{x}, \mathbf{x}^{\prime}\right)+\sum_{n=1}^{N} \theta_{n}\left(\mathbf{x}, \mathbf{x}^{\prime}\right) y_{n}
$$

We assume that $\theta_{0}\left(\mathbf{x}, \mathbf{x}^{\prime}\right)>C>0$ for some constant $C$ that is sufficiently large so that, for all $\boldsymbol{y} \in \Gamma, \theta\left(\mathbf{x}, \mathbf{x}^{\prime} ; \boldsymbol{y}\right)$ satisfies $(9)$; we also assume that $\theta\left(\mathbf{x}, \mathbf{x}^{\prime} ; \boldsymbol{y}\right)=\theta\left(\mathbf{x}^{\prime}, \mathbf{x} ; \boldsymbol{y}\right)$. Such an affine dependence arises, e.g., for truncated Karhunen-Loève expansions of a (correlated) random field $\theta\left(\mathbf{x}, \mathbf{x}^{\prime} ; \boldsymbol{y}\right)$ [35]. Then, for any $\boldsymbol{y} \in \Gamma$, a weak formulation of the problem (8) is given by: seek $u(\cdot, \boldsymbol{y}) \in \mathcal{V}_{c}\left(D \cup D_{I}\right)$ such that

$$
a(u, v ; \boldsymbol{y})=(f, v) \quad \forall v \in \mathcal{V}_{c}
$$

where

$$
a(u, v ; \boldsymbol{y})=a_{0}(u, v)+\sum_{n=1}^{N} y_{n} a_{n}(u, v) \quad \forall \boldsymbol{y} \in \Gamma, u, v \in \mathcal{V}_{c}\left(D \cup D_{I}\right) .
$$

Here, for $n=0, \ldots, N$ and for all $u, v \in \mathcal{V}_{c}\left(D \cup D_{I}\right)$,

$$
a_{n}(u, v)=\frac{1}{2} \int_{D \cup D_{I}} \int_{D \cup D_{I}} \gamma\left(\mathbf{x}, \mathbf{x}^{\prime}\right) \theta_{n}\left(\mathbf{x}, \mathbf{x}^{\prime}\right)\left(u(\mathbf{x})-u\left(\mathbf{x}^{\prime}\right)\right)\left(v(\mathbf{x})-v\left(\mathbf{x}^{\prime}\right)\right) d \mathbf{x} d \mathbf{x}^{\prime}
$$

Note that for all $n=0, \ldots, N, a_{n}(u, v)$ do not depend on $\boldsymbol{y}$.

For spatial discretization, we use finite element methods. We denote by $V_{h}^{f e}$ the finite element space of continuous piecewise polynomials defined with respect to a regular triangulation of $D$ and denote by $J$ the dimension of $V_{h}^{f e}$. For any $\boldsymbol{y} \in \Gamma$, the finite element approximation $u_{h}(\mathbf{x}, \boldsymbol{y}) \in V_{h}^{f e} \subset \mathcal{V}_{c}\left(D \cup D_{I}\right)$ of the solution $u(\mathbf{x}, \boldsymbol{y})$ of (12) is determined by solving

$$
a\left(u_{h}(\cdot, \boldsymbol{y}), v ; \boldsymbol{y}\right)=(f(\cdot, \boldsymbol{y}), v) \quad \forall v \in V_{h}^{f e} .
$$

For any $\boldsymbol{y} \in \Gamma,(7)$ provides and estimate for the error in the piecewise linear finite element approximation of the exact solution of (12).

\section{A reduced-basis method}

In our context, a reduced-basis method collects deterministic solutions of the nonlocal problem at several points in $\Gamma$, then uses these solutions as a basis to approximate solutions at other points in $\Gamma$ through a Galerkin projection. We now describe the specific reducedbasis method we use. We assume that $\theta\left(\mathbf{x}, \mathbf{x}^{\prime} ; \boldsymbol{y}\right)$ can be expressed in the form of (11) so that we have the weak formulation given in (12) defining the exact solution $u(\mathbf{x}, \boldsymbol{y})$ and the discrete weak formulation (14) defining the finite element approximation $u_{h}(\mathbf{x}, \boldsymbol{y})$.

The construction of the reduced-basis method begins by choosing a training set $\Xi_{\text {train }}$ of $M_{\text {train }}$ points (e.g., $\left.M_{\text {train }}=1000\right)$ in $\Gamma$; these points could be chosen randomly according 
to the joint $\operatorname{PDF} \rho(\boldsymbol{y})$ associated with the random parameters $\boldsymbol{y} \in \Gamma$. A subset $\Xi_{k}=$ $\left\{\boldsymbol{y}_{i}\right\}_{i=0}^{k-1} \subset \Xi_{\text {train }}$ such that $1 \leq k \ll J$ of the points in $\Xi_{\text {train }}$ is then chosen; $k=1$ is the usual choice and the particular point chosen is usually somewhere near the center of $\Gamma$. We then solve the spatially discretized PDE (14) $k$ times to obtain the set of $k$ finite element approximations $\left\{u_{h}^{i}(\mathbf{x})=u_{h}\left(\mathbf{x}, \boldsymbol{y}_{i}\right)\right\}_{i=0}^{k-1}$; using these solutions, we define the space $V_{k}^{r b}=\operatorname{span}\left\{u_{h}^{i}(\mathbf{x})\right\}_{i=0}^{k-1} \subset V_{h}^{f e}$. Using a Galerkin method, we then find a reduced-basis approximate solution $u_{h, k}(\mathbf{x}, \boldsymbol{y})$ of (12), i.e., we solve the following problem: seek

$$
u_{h, k}(\mathbf{x})=\sum_{i=0}^{k-1} c_{i}^{k}(\boldsymbol{y}) u_{h}^{i}(\mathbf{x}) \in V_{k}^{r b}
$$

satisfying

$$
a\left(u_{h, k}, v ; \boldsymbol{y}\right)=(f, v) \quad \forall v \in V_{k}^{r b},
$$

where $a(u, v ; \boldsymbol{y})$ is defined in (13). Note that, for each $\boldsymbol{y} \in \Gamma,(16)$ is equivalent to a linear system of $k$ algebraic equations for the coefficients $\left\{c_{i}^{k}(\boldsymbol{y})\right\}_{i=0}^{k-1}$ in (15).

To measure the error between the finite element solution at any point $\boldsymbol{y} \in \Gamma$ and the reduced-basis solution at that point, we use the norm

$$
\|v\|,^{2}=\frac{1}{2} \int_{D \cup D_{I}} \int_{D \cup D_{I}} \gamma\left(\mathbf{x}, \mathbf{x}^{\prime}\right)\left(v(\mathbf{x})-v\left(\mathbf{x}^{\prime}\right)\right)^{2} d \mathbf{x} d \mathbf{x}^{\prime}
$$

for $v \in \mathcal{V}_{c}\left(D \cup D_{I}\right)$. Corresponding to this norm we have the inner product

$$
((u, v))=\frac{1}{2} \int_{D \cup D_{I}} \int_{D \cup D_{I}} \gamma\left(\mathbf{x}, \mathbf{x}^{\prime}\right)\left(u(\mathbf{x})-u\left(\mathbf{x}^{\prime}\right)\right)\left(v(\mathbf{x})-v\left(\mathbf{x}^{\prime}\right)\right) d \mathbf{x} d \mathbf{x}^{\prime},
$$

i.e., $\|v\|^{2}=((v, v))$. It is easy to verify that this norm is equivalent to the norm $\|\cdot\|_{\mathcal{V}_{c}}$ defined in $§ 1.1$. Note that in relation to the "energy" norm $\|u\|_{\mathcal{V}_{c}}$, we have

$$
\theta_{\min }\left|\|v\|\left\|^{2} \leq \underline{\theta}(\boldsymbol{y})\right\| v\right|\left\|^{2} \leq a(v, v ; \boldsymbol{y}) \leq \bar{\theta}(\boldsymbol{y})\right\| v\left\|^{2} \leq \theta_{\max }\right\| v\|\|^{2},
$$

where $v \in \mathcal{V}_{c}\left(D \cup D_{I}\right)$, and for any $\boldsymbol{y} \in \Gamma, \underline{\theta}(\boldsymbol{y})=\min _{\mathbf{x}, \mathbf{x}^{\prime} \in D \cup D_{I}} \theta\left(\mathbf{x}, \mathbf{x}^{\prime}, \boldsymbol{y}\right), \bar{\theta}(\boldsymbol{y})=$ $\max _{\mathbf{x}, \mathbf{x}^{\prime} \in D \cup D_{I}} \theta\left(\mathbf{x}, \mathbf{x}^{\prime}, \boldsymbol{y}\right)$.

The next step is to "improve" the initial point set $\Xi_{k}=\left\{\boldsymbol{y}_{i}\right\}_{i=0}^{k-1}$. By this we mean to add a point $\boldsymbol{y}_{k}$ to the set $\Xi_{k}$ to produce the point set $\Xi_{k+1}=\left\{\boldsymbol{y}_{i}\right\}_{i=0}^{k}$ of $k+1$ points. Ideally, the new point $\boldsymbol{y}_{k}$ is chosen by the greedy algorithm

$$
\boldsymbol{y}_{k}=\arg \sup _{\boldsymbol{y} \in \Gamma}\left\|u_{h, k}(\cdot, \boldsymbol{y})-u_{h}(\cdot, \boldsymbol{y})\right\| \|
$$

i.e., the point in $\Gamma$ at which the error in the current reduced-basis approximation relative to the finite element solution is largest. We then add the corresponding finite element solution $u_{h}^{k}(\mathbf{x})=u_{h}\left(\mathbf{x}, \boldsymbol{y}_{k}\right)$ to the other solutions to define the new reduced-basis space $V_{k+1}^{r b}=\operatorname{span}\left\{u_{h}^{i}(\mathbf{x})\right\}_{i=0}^{k} \subset V_{h}^{f e}$. 
Now that we know how to improve a point set, we can continue repeating the process of enhancing the reduced basis, until the difference between the reduced-basis and full finite element solutions satisfy some convergence criteria. Determining the point $\boldsymbol{y}_{k}$ by solving the optimization problem (19) requires discretization. Here we use

$$
\boldsymbol{y}_{k}=\arg \max _{\boldsymbol{y} \in \Xi_{\text {train }}}\left\|u_{h, k}(\cdot, \boldsymbol{y})-u_{h}(\cdot, \boldsymbol{y})\right\| .
$$

Because the initial point set $\Xi_{k}$ is chosen from the training set, i.e., $\Xi_{k} \subset \Xi_{\text {train }}$, obviously $\Xi_{k+1} \subset \Xi_{\text {train }}$ as well. Unfortunately, even (20) is not a practical procedure because it requires $M_{\text {train }}$ expensive finite element solves. We do not want to do this because our goal is to avoid finite element solves whenever possible. Thus, instead, we use residuals determined from the reduced-basis solution to determine $\boldsymbol{y}_{k}$. In the next section, we describe this procedure.

\subsection{Implementation of the weak greedy algorithm}

Suppose that after $k$ steps of the greedy algorithm we have determined a set of $k \geq 1$ points $\Xi_{k}$ and the corresponding reduced-basis space $V_{k}^{r b}=\operatorname{span}\left\{u_{h}^{i}(\mathbf{x})=u_{h}\left(\mathbf{x}, \boldsymbol{y}_{i}\right)\right\}_{i=0}^{k-1} \subset$ $V_{h}^{f e}$ with $\boldsymbol{y}_{i} \in \Xi_{k}$. Then, for any $\boldsymbol{y} \in \Gamma$, we can obtain the coefficients $c_{i}^{k}(\boldsymbol{y})$ and $u_{h, k}(\mathbf{x}, \boldsymbol{y})=\sum_{i=0}^{k-1} c_{i}^{k}(\boldsymbol{y}) u_{h}^{i}(\mathbf{x}) \in V_{k}^{r b}$ by solving the Galerkin discrete system

$$
a\left(u_{h, k}, v ; \boldsymbol{y}\right)=(f, v) \quad \forall v \in V_{k}^{r b} .
$$

Then, we start the $(k+1)$ st step by firstly giving the error between $u_{h}(\mathbf{x}, \boldsymbol{y}) \in V_{h}^{f e}$ and its Galerkin projection $u_{h, k}(\mathbf{x}, \boldsymbol{y})$ in $V_{k}^{r b}$. Letting $e_{h, k}(\mathbf{x}, \boldsymbol{y})=u_{h}(\mathbf{x}, \boldsymbol{y})-u_{h, k}(\mathbf{x}, \boldsymbol{y}) \in V_{h}^{f e}$ and using (14), we have that, for any $\boldsymbol{y} \in \Gamma$,

$$
a\left(e_{h, k}(\cdot, \boldsymbol{y}), v ; \boldsymbol{y}\right)=(f, v)-a\left(u_{h, k}(\cdot, \boldsymbol{y}), v ; \boldsymbol{y}\right) \quad \forall v \in V_{h}^{f e} .
$$

The right-hand side of (22) can be viewed as the residual corresponding to the reducedorder solution $u_{h, k}(\mathbf{x}, \boldsymbol{y})$ with respect to the finite element space $V_{h}^{f e}$. Next, for any $\boldsymbol{y} \in \Gamma$, we define $\widehat{e}_{h, k}(\mathbf{x}, \boldsymbol{y})$ by

$$
\begin{aligned}
\left(\left(\widehat{e}_{h, k}(\cdot, \boldsymbol{y}), v\right)\right) & =a\left(e_{h, k}(\cdot, \boldsymbol{y}), v ; \boldsymbol{y}\right) \\
& =(f, v)-a\left(u_{h, k}(\cdot, \boldsymbol{y}), v ; \boldsymbol{y}\right) \quad \forall v \in V_{h}^{f e} .
\end{aligned}
$$

With $a(u, v ; \boldsymbol{y})$ given by (13), we then have that, for all $v \in V_{h}^{f e}$,

$$
\begin{aligned}
& \left(\left(\widehat{e}_{h, k}(\cdot, \boldsymbol{y}), v\right)\right)=(f, v)-a_{0}\left(u_{h, k}(\cdot, \boldsymbol{y}), v\right)+\sum_{n=1}^{N} y_{n} a_{n}\left(u_{h, k}(\cdot, \boldsymbol{y}), v\right) \\
& \quad=(f, v)-a_{0}\left(\sum_{i=0}^{k-1} c_{i}^{k}(\boldsymbol{y}) u_{h}^{i}(\cdot), v\right)+\sum_{n=1}^{N} y_{n} a_{n}\left(\sum_{i=0}^{k-1} c_{i}^{k}(\boldsymbol{y}) u_{h}^{i}(\cdot), v\right) \\
& =(f, v)-\sum_{i=0}^{k-1} c_{i}^{k}(\boldsymbol{y})\left(a_{0}\left(u_{h}^{i}(\mathbf{x}), v\right)+\sum_{n=1}^{N} y_{n} a_{n}\left(u_{h}^{i}(\mathbf{x}), v\right)\right) .
\end{aligned}
$$


One can determine $\widehat{e}_{h, k}(\mathbf{x}, \boldsymbol{y}) \in V_{h}^{f e}$ efficiently by solving the problems

$$
\left\{\begin{array}{l}
\left(\left(\widehat{e}^{f}, v\right)\right)=(f, v) \quad \forall v \in V_{h}^{f e} \\
\left(\left(\widehat{e}_{i}^{n}, v\right)\right)=a_{n}\left(u_{h}^{i}, v\right) \quad \forall v \in V_{h}^{f e} \quad \text { for } i=0, \ldots, k-1 \text { and } n=0, \ldots, N
\end{array}\right.
$$

However, note that the problem for $\widehat{e}^{f}(\mathbf{x})$ is independent of $i$ so that it can be solved during the initialization of the greedy algorithm. For $k=1$, the second part of system (25) needs to be solved once. For $k \geq 2$, the problems for $\widehat{e}_{i}^{n}(\mathbf{x})$ for $i=0, \ldots, k-2$ and $n=0, \ldots, N$ have already been solved in previous steps. Thus, at this $(k+1)$ th step of the greedy algorithm we need only solve the $N+1$ problems

$$
\left(\left(\widehat{e}_{k-1}^{n}, v\right)\right)=a_{n}\left(u_{h}^{k-1}, v\right) \quad \forall v \in V_{h}^{f e} \quad n=0, \ldots, N
$$

which do not involve $\boldsymbol{y}$. Substituting (25) into (24), we obtain, for any $\boldsymbol{y} \in \Gamma$,

$$
\left(\left(\widehat{e}_{h, k}(\cdot, \boldsymbol{y}), v\right)\right)=\left(\left(\widehat{e}^{f}(\cdot), v\right)\right)-\sum_{i=0}^{k-1} c_{i}^{k}(\boldsymbol{y})\left(\left(\left(\widehat{e}_{i}^{0}(\cdot), v\right)\right)+\sum_{n=1}^{N} y_{n}\left(\left(\widehat{e}_{i}^{n}(\cdot), v\right)\right)\right) .
$$

Because $\widehat{e}_{h, k}, \widehat{e}^{f}$, and all of $\widehat{e}_{i}^{n}(\cdot)$ belong to $V_{h}^{f e}$, we then have that

$$
\widehat{e}_{h, k}(\mathbf{x}, \boldsymbol{y})=\widehat{e}^{f}(\mathbf{x})-\sum_{i=0}^{k-1} c_{i}^{k}(\boldsymbol{y})\left(\widehat{e}_{i}^{0}(\mathbf{x})+\sum_{n=1}^{N} y_{n} \widehat{e}_{i}^{n}(\mathbf{x})\right) \in V_{h}^{f e}
$$

Using (18) and (23) and the definition of the norms, we have

$$
\begin{aligned}
\underline{\theta}(\boldsymbol{y})\left\|e_{h, k}(\cdot, \boldsymbol{y})\right\|^{2} & \leq\left\|e_{h, k}(\cdot, \boldsymbol{y})\right\|_{\mathcal{V}_{c}}^{2}=a\left(e_{h, k}(\cdot, \boldsymbol{y}), e_{h, k}(\cdot, \boldsymbol{y}) ; \boldsymbol{y}\right) \\
& =\left(\left(\widehat{e}_{h, k}(\cdot, \boldsymbol{y}), e_{h, k}(\cdot, \boldsymbol{y})\right)\right) \leq\left\|\widehat{e}_{h, k}(\cdot, \boldsymbol{y})\right\|\|\| e_{h, k}(\cdot, \boldsymbol{y})\|\|
\end{aligned}
$$

so that, for any $\boldsymbol{y} \in \Gamma$, we have that

$$
\left\|u_{h}(\cdot, \boldsymbol{y})-u_{h, k}(\cdot, \boldsymbol{y})\right\|=\left\|e_{h, k}(\cdot, \boldsymbol{y})\right\| \leq \frac{1}{\underline{\theta}(\boldsymbol{y})}\left\|\widehat{e}_{h, k}(\cdot, \boldsymbol{y})\right\| \| .
$$

Similarly, by (18) and (23), we have

$$
\left\|\widehat{e}_{h, k}(\cdot, \boldsymbol{y})\right\|\left\|=a\left(e_{h, k}(\cdot, \boldsymbol{y}), \widehat{e}_{h, k}(\cdot, \boldsymbol{y}) ; \boldsymbol{y}\right) /\right\| \widehat{e}_{h, k}(\cdot, \boldsymbol{y})\|\leq \bar{\theta}(\boldsymbol{y})\| e_{h, k}(\cdot, \boldsymbol{y})\|\| .
$$

Then,

$$
\left\|e_{h, k}(\cdot, \boldsymbol{y})\right\| \leq \frac{1}{\underline{\theta}(\boldsymbol{y})}\left\|\widehat{e}_{h, k}(\cdot, \boldsymbol{y})\right\| \mid \leq \frac{\bar{\theta}(\boldsymbol{y})}{\underline{\theta}(\boldsymbol{y})}\left\|e_{h, k}(\cdot, \boldsymbol{y})\right\|
$$

which means

$$
C \max _{\boldsymbol{y} \in \Xi_{\text {train }}}\left\{\frac{1}{\underline{\theta}(\boldsymbol{y})}\left\|\widehat{e}_{h, k}(\cdot, \boldsymbol{y})\right\|\right\} \leq \max _{\boldsymbol{y} \in \Xi_{\text {train }}}\left\|e_{h, k}(\cdot, \boldsymbol{y})\right\| \|, \leq \max _{\boldsymbol{y} \in \Xi_{\text {train }}}\left\{\frac{1}{\underline{\theta}(\boldsymbol{y})}\left\|\widehat{e}_{h, k}(\cdot, \boldsymbol{y})\right\|\right\},
$$


where $C=\theta_{\min } / \theta_{\max }$. We determine the next point $\boldsymbol{y}_{k}$ by

$$
\boldsymbol{y}_{k}=\arg \max _{\boldsymbol{y} \in \Xi_{\text {train }}}\left\{\frac{1}{\underline{\theta}(\boldsymbol{y})}\left\|\widehat{e}_{h, k}(\cdot, \boldsymbol{y})\right\|\right\} .
$$

We then define the new point set $\Xi_{k+1}=\Xi_{k} \cup \boldsymbol{y}_{k}$ and proceed to solve the finite element system $a\left(u_{h}\left(\cdot, \boldsymbol{y}_{k}\right), v ; \boldsymbol{y}_{k}\right)=(f, v)$ for all $v \in V_{h}^{f e}$ to determine the new reduced-basis function $u_{h}^{k}(\mathbf{x})=u_{h}\left(\mathbf{x}, \boldsymbol{y}_{k}\right) \in V_{h}^{f e}$ and to define the new reduced-basis space $V_{k+1}^{r b}=$ $\operatorname{span}\left\{u_{h}^{i}(\mathbf{x})=u_{h}\left(\mathbf{x}, \boldsymbol{y}_{i}\right)\right\}_{i=0}^{k} \subset V_{h}^{f e}$. At this point, the step of the greedy algorithm is completed. The greedy algorithm can be terminated whenever

$$
\max _{\boldsymbol{y} \in \Xi_{\text {train }}}\left\{\frac{1}{\underline{\theta}(\boldsymbol{y})}\left\|\widehat{e}_{h, r}(\cdot, \boldsymbol{y})\right\|\right\} \leq \tau
$$

for a prescribed tolerance $\tau>0$. From, (27), this guarantees that

$$
\max _{\boldsymbol{y} \in \Xi_{\text {train }}}\left\|u_{h}(\cdot, \boldsymbol{y})-u_{h, k}(\cdot, \boldsymbol{y})\right\|=\max _{\boldsymbol{y} \in \Xi_{\text {train }}}\left\|e_{h, k}(\cdot, \boldsymbol{y})\right\| \leq \tau
$$

as well.

Before proceeding, we note that the reduced-basis $V_{k}^{r b}=\operatorname{span}\left\{u_{h}^{i}(\mathbf{x})\right\}_{i=0}^{k-1} \subset V_{h}^{f e}$ can be ill conditioned, increasingly so as $k$ increases. Thus, in the implementation of the greedy algorithm, we apply a modified Gram-Schmidt method [34] to determine and equivalent orthonormal basis. Specifically, if after $k$ steps of the greedy algorithm we have the orthonormal basis $\left\{\xi_{h}^{i}(\mathbf{x})\right\}_{i=0}^{k-1}$ for $V_{k}^{r b}$, then we apply the Gram-Schmidt method to the basis $\left\{\xi_{h}^{i}(\mathbf{x})\right\}_{i=0}^{k-1} \cup u_{h}^{k}(\mathbf{x})$ to determine $\xi_{h}^{k}(\mathbf{x})$ such that $\left\{\xi_{h}^{i}(\mathbf{x})\right\}_{i=0}^{k} \subset V_{h}^{f e}$ is an orthonormal basis for $V_{k+1}^{r b}$.

A final remark explains why we introduce, in $(23), \widehat{e}_{h, k}(\mathbf{x}, \boldsymbol{y})$ instead of working directly with $e_{h, k}(\mathbf{x}, \boldsymbol{y})$. If we had done the latter, the problems we would have to solve to determine $e_{h, k}(\mathbf{x}, \boldsymbol{y})$ would involve $a(\cdot, v ; \boldsymbol{y})$ instead of $((\cdot, v))$; the latter is independent of $\boldsymbol{y}$ whereas the former explicitly depends on $\boldsymbol{y}$ through the appearance of $\theta\left(\mathbf{x}, \mathbf{x}^{\prime} ; \boldsymbol{y}\right)$, a fact that would prevent the efficient implementation of the greedy algorithm.

\subsection{Convergence analysis}

As in [41], we define $\mathcal{F}:=\left\{u_{h}(\mathbf{x}, \boldsymbol{y}), \boldsymbol{y} \in \Xi_{\text {train }}\right\}$ and the distance is measured by $\|\cdot \mid\|$, and let

$$
\sigma_{k}:=\sup _{f \in \mathcal{F}} \inf _{v_{k} \in V_{k}^{r b}} \operatorname{dist}\left(f, v_{k}\right), \quad d_{k}:=\inf _{Y_{k}} \sup _{f \in \mathcal{F}} \operatorname{dist}\left(f, Y_{k}\right),
$$

where $V_{k}^{r b}$ is $k$ dimensional reduced-basis subspace of $\mathcal{F}, \sigma_{k}$ is the distance indicating how well $\mathcal{F}$ can be approximated by $V_{k}^{r b} ; Y_{k}$ is $n$ dimensional subset of $V_{h}^{f e}, d_{k}$ is the Kolmogorov width of $\mathcal{F}$. For the weak greedy algorithm, at the first step one can choose a function $u_{h}\left(\cdot, \boldsymbol{y}_{0}\right) \in \mathcal{F}$ which is not zero and normalize it. The proof of Corollary 3.3 in [41] is still valid. After $V_{k}^{r b}=\left\{u_{h}^{0}, \cdots, u_{h}^{k-1}\right\}$ is chosen, the next basis $u_{h}^{k}$ is determined by (28). Combined with (27), we have

$$
\operatorname{dist}\left(u_{h}\left(\cdot, \boldsymbol{y}_{k}\right), V_{k}^{r b}\right) \geq \gamma \sigma_{k}, \gamma=\frac{\theta_{\min }}{\theta_{\max }}
$$


so that by Corollary 3.3 in [41], we have

$$
\sigma_{2 k} \leq \sqrt{2} \gamma^{-1} \sqrt{d_{k}}, k=1,2, \ldots
$$

Define next

$$
e:=\sup _{v_{h} \in V_{h}} \inf _{f \in \mathcal{F}} \operatorname{dist}\left(v_{h}, f\right), V_{h}=\left\{u_{h}(\cdot, \boldsymbol{y}), \boldsymbol{y} \in \Gamma\right\} .
$$

Because at each point $\boldsymbol{y} \in \Gamma, u_{h, 2 k}(\cdot, \boldsymbol{y})$ is the Galerkin projection of $u_{h}(\cdot, \boldsymbol{y})$ in $V_{2 k}^{r b}$, then for $v \in V_{2 k}^{r b}$ and $f \in \mathcal{F}$

$$
\begin{aligned}
\frac{\theta_{\min }}{\theta_{\max }}\left\|u_{h, 2 k}(\cdot, \boldsymbol{y})-u_{h}(\cdot, \boldsymbol{y})\right\| & \leq\left\|v-u_{h}(\cdot, \boldsymbol{y})\right\| \\
& \leq\|v-f\|+\left\|f-u_{h}(\cdot, \boldsymbol{y})\right\| \\
& \leq \sup _{f \in \mathcal{F}} \inf _{v \in V_{2 k}^{r b}}\|v-f\|+\sup _{v_{h} \in V_{h}} \inf _{f \in \mathcal{F}}\left\|f-v_{h}\right\| \\
& \leq \sigma_{2 k}+e \leq \sqrt{2} \gamma^{-1} \sqrt{d_{k}}+e
\end{aligned}
$$

which means

$$
\sup _{\boldsymbol{y} \in \Gamma}\left\|u_{h, 2 k}(\cdot, \boldsymbol{y})-u_{h}(\cdot, \boldsymbol{y})\right\| \leq \sqrt{2} \gamma^{-2} \sqrt{d_{k}}+\gamma^{-1} e
$$

It is easy to see

$$
\sup _{\boldsymbol{y} \in \Gamma}\left\|u_{h, 2 k}(\cdot, \boldsymbol{y})-u(\boldsymbol{y})\right\| \leq \sqrt{2} \gamma^{-2} \sqrt{d_{k}}+\gamma^{-1} e+\sup _{\boldsymbol{y} \in \Gamma}\left\|u_{h}(\cdot, \boldsymbol{y})-u(\cdot, \boldsymbol{y})\right\|,
$$

where $u(\cdot, \boldsymbol{y}) \in C^{0}(\Gamma ; V)$, so that the sum on the right-hand side decreases to 0 if $h \rightarrow 0$, $k \rightarrow J$, and $\Xi_{\text {train }}$ becomes denser in $\Gamma$. Generally speaking, $e$ is much smaller than $d_{k}$. Suppose the assumption $d_{k} \leq C \exp \left(-c k^{\alpha}\right)$ is correct. Then, for $C_{1} \exp \left(-c_{1} k^{\alpha}\right)>e$

$$
\sup _{\boldsymbol{y} \in \Gamma}\left\|u_{h, 2 k}(\boldsymbol{y})-u_{h}(\boldsymbol{y})\right\| \leq 2 C_{1} \exp \left(-c_{1} k^{\alpha}\right),
$$

so that we have sub-exponential convergence rate for the error which is similar to our numerical results.

\section{A simplified reduced-basis method}

A simplified reduced-basis method was studied in [30]. Here, we state the main differences compared with the algorithm of $\S 3.1$. Similarly, suppose that we have $V_{k}^{r b}$ and the (21); then the matrix form of (21) is

$$
U^{T} A_{\boldsymbol{y}} U c_{\boldsymbol{y}}^{k}=U^{T} F
$$

where $\boldsymbol{y}=\left(y_{1}, \cdots, y_{N}\right), c_{\boldsymbol{y}}^{k}=\left(c_{0}^{k}(\boldsymbol{y}), \cdots, c_{k-1}^{k}(\boldsymbol{y})\right)^{T}$, and $F=\left(\left(f, \phi_{1}\right), \cdots,\left(f, \phi_{J}\right)\right)^{T}$. Also, as

$$
u_{h}^{i}=\sum_{j=1}^{J} u_{j}^{i} \phi_{j}, \quad i=0, \ldots, k-1
$$


we define

$$
U=\left(U_{0}, \cdots, U_{k-1}\right), \text { where } U_{i}=\left(u_{1}^{i}, u_{2}^{i}, \cdots, u_{J}^{i}\right)^{T}
$$

and

$$
A_{\boldsymbol{y}}=A_{0}+\sum_{n=1}^{N} y_{n} A_{n}, \text { where } A_{n}(i, j)=a_{n}\left(\phi_{i}, \phi_{j}\right), \quad n=0, \ldots, N .
$$

For this method, instead of $e_{h, k}$ in $§ 3.1$, we define a simpler error

$$
\begin{aligned}
e_{\boldsymbol{y}} & =A_{\boldsymbol{y}} U c_{\boldsymbol{y}}^{k}-F \\
& =\sum_{i=1}^{k} c_{i-1}^{k}(\boldsymbol{y})\left(\sum_{q=1}^{N} y_{i} A_{q} U+A_{0} U\right)-F .
\end{aligned}
$$

Instead of (28), the next point $\boldsymbol{y}_{k}$ is determined by

$$
\boldsymbol{y}_{k}=\arg \max _{\boldsymbol{y} \in \Xi_{\text {train }}}\left\{\left\|e_{\boldsymbol{y}}\right\|_{2} /\|F\|_{2}\right\},
$$

where $\|\cdot\|_{2}$ is the vector 2 norm. The computational cost of simplified reduced-basis method is smaller than the method of $\S 3.1$.

\section{Numerical results}

In this section, we illustrate the costs of using the reduced basis methods of $\S 3$ and $\S 4$ relative to the costs of using full finite element solves. We use the total number of flops as the measure of computational costs.

\subsection{Setup}

We consider the one-dimensional nonlocal diffusion problem

$$
\left\{\begin{array}{l}
\frac{2-2 s}{\delta^{2-2 s}} \int_{x-\delta}^{x+\delta} \frac{\theta\left(x, x^{\prime} ; \boldsymbol{y}\right)\left(u(x, \boldsymbol{y})-u\left(x^{\prime}, \boldsymbol{y}\right)\right)}{\left|x-x^{\prime}\right|^{1+2 s}} d x^{\prime}=1 \quad \text { for } x \in(0,1), \boldsymbol{y} \in \Gamma, \\
u(x, \boldsymbol{y})=0 \text { for } x \in[-\delta, 0] \cup[1,1+\delta], \boldsymbol{y} \in \Gamma,
\end{array}\right.
$$

where the random variables $\boldsymbol{y}=\left(y_{1}, \cdots, y_{N}\right)$ are uniformly distributed in $\Gamma=[-\sqrt{3}, \sqrt{3}]^{N}$,

$\theta\left(x, x^{\prime}, \boldsymbol{y}\right)=\frac{1}{2}\left(a(x, \boldsymbol{y})+a\left(x^{\prime}, \boldsymbol{y}\right)\right)$, and we have the truncated Karhunen-Loève expansion

$$
a(x, \boldsymbol{y})=\frac{1}{100}\left(\widehat{a}+\left(\frac{\sqrt{\pi} S}{2}\right)^{1 / 2} y_{1}+\sum_{n=2}^{N} \phi_{n}(x) y_{n}\right)
$$

with

$$
\phi_{n}(x)= \begin{cases}\sqrt{\lambda_{n}} \sin \left(\frac{n}{2} \pi x\right), & \text { for } n \text { even } \\ \sqrt{\lambda_{n}} \cos \left(\frac{n-1}{2} \pi x\right), & \text { for } n \text { odd }\end{cases}
$$


and

$$
\sqrt{\lambda_{n}}=(\sqrt{\pi} S)^{1 / 2} \exp \left(-\frac{1}{32}((n-\bmod (n, 2)) \pi S)^{2}\right) .
$$

The constant $\widehat{a}$ is chosen sufficiently large so that $a(x, \boldsymbol{y})$ is bounded away from zero for all $x$ and $\boldsymbol{y}$. The constant $S$ denotes the correlation length of the random field. Let $V_{h}^{f e}$ denote the space of piecewise linear polynomials constructed with respect to a uniform partition of the spatial domain $D \cup D_{I}=(-\delta, 1+\delta)$ into subintervals of length $h$ such that there are exactly $J$ interior nodes in the solution domain $D=(0,1)$. We also set to zero the nodal values of functions belonging to $V_{h}^{f e}$ corresponding to the grid points in $D_{I}=[-\delta, 0] \cup[1,1+\delta]$ so that $V_{h}^{f e} \subset \mathcal{V}_{c}\left(D \cup D_{I}\right)$ and the dimension of $V_{h}^{f e}$ is $J$.

For any $\boldsymbol{y} \in \Gamma$, the solution of the finite element problem in (14) can be obtained by solving a linear system $A u=f$, where $A_{i j}=a\left(\phi_{i}, \phi_{j} ; \boldsymbol{y}\right)$ and $f_{i}=\left(f, \phi_{i}\right)$. It is known that the sparsity of the matrix $A$ deteriorates as the value of horizon $\delta$ increases. In this one-dimensional example, the bandwidth of matrix $A$ is naturally minimized, so that we can use the banded Cholesky decomposition method to solve the linear system efficiently; see [39]. The costs are evaluated by flops, and,,$+- \times, \div, \sqrt{ }$ are treated equally, each operation counts once. Noted that although the banded Cholesky solver is not the best choice for this problem, it can help illustrate the advantages of the proposed reduced-basis method. Moreover, all the conclusions drawn from the numerical results below will carry over to the case of using efficient iterative solvers for more complicated multidimensional nonlocal problems.

Here we have three ways to define an approximation of the solution $u(x, \boldsymbol{y})$, i.e.,

- RB1: the reduced basis solution $u_{r b 1}(x, \boldsymbol{y}) \in V_{h}^{f e}$ using the algorithm in $\S 3$;

- RB2: the reduced basis solution $u_{r b 2}(x, \boldsymbol{y}) \in V_{h}^{f e}$ using the algorithm in $\S 4$;

- FEM: the sparse grid interpolant ${ }^{2} u_{s g}(x, \boldsymbol{y})$ using the finite element solution $u_{h}(x, \boldsymbol{y}) \in$ $V_{h}^{f e}$ given in (14).

The error measures we use are based on the norm

$$
\|v\|_{h}^{2}=a_{0}(v, v)=\frac{2-2 s}{\delta^{2-2 s}} \int_{0}^{1} \int_{0}^{1} \frac{\left(v(x)-v\left(x^{\prime}\right)\right)^{2}}{\left|x-x^{\prime}\right|^{1+2 s}} \chi_{\left|x-x^{\prime}\right| \leq \delta} d x^{\prime} d x \quad \forall v \in V_{h}^{f e} .
$$

Given a set $\Xi_{\text {test }} \subset \Gamma$ of $M_{\text {test }}$ randomly selected points in $\Gamma$, we then define the error measures

$$
\varepsilon_{\infty}=\max _{\boldsymbol{y} \in \Xi_{\text {test }}}\left\|u_{h}-u_{\text {surrogate }}\right\|_{h}
$$

and

$$
\varepsilon_{\text {ave }}=\left\|\frac{1}{M_{\text {test }}} \sum_{\boldsymbol{y} \in \Xi_{\text {test }}}\left(u_{h}-u_{\text {surrogate }}\right)\right\| \|_{h}
$$

\footnotetext{
${ }^{2} \mathrm{~A}$ brief introduction to the sparse grid interpolation is given in Appendix A.
} 
where, for any $\boldsymbol{y} \in \Xi_{\text {test }} \subset \Gamma, u_{h}(x, \boldsymbol{y}) \in V_{h}^{f e}$ denotes the finite element solution, and $u_{\text {surrogate }}(x, \boldsymbol{y}) \in V_{h}^{f e}$ denotes any of the three surrogates defined above. Note that the points in $\Xi_{\text {test }}$ are different from those in the training set $\Xi_{\text {train }}$ used in the construction of the reduced basis and are also different from the sparse grid points $\Xi_{\mathrm{sg}}$ used to construct the sparse-grid interpolants. For the examples in $\S 5.3$ and $\S 5.4$, the training set $\Xi_{\text {train }}$ (used in the construction of the reduced bases) and the test set $\Xi_{\text {test }}$ (used in the error measures) both consists of $M_{\text {train }}=1000$ and $M_{\text {test }}=1000$ randomly selected points in $\Gamma$, respectively; of course, the two point sets are chosen independently of each other.

\subsection{Convergence to classic local diffusion problems}

It is known (see, e.g., [40]) that the nonlocal problem (32) reduces to the (local) differential equation problem

$$
\left\{\begin{array}{l}
-\frac{\mathrm{d}}{\mathrm{d} x}\left(a(x, \boldsymbol{y}) \frac{\mathrm{d}}{\mathrm{d} x} u^{l o c}(x, \boldsymbol{y})\right)=1 \quad \text { for } x \in(0,1), \boldsymbol{y} \in \Gamma \quad \text { as } \delta \rightarrow 0 \\
u^{l o c}(0, \boldsymbol{y})=u^{l o c}(1, \boldsymbol{y})=0 \quad \text { for } \boldsymbol{y} \in \Gamma
\end{array}\right.
$$

and also that, as $\delta \rightarrow 0$, the solution $u(\cdot, \boldsymbol{y})$ of the nonlocal problem (32) converges to the solution $u^{l o c}(\cdot, \boldsymbol{y})$ of the local problem (36). To illustrate these results, we measure the difference between the finite element approximation $u_{h}^{l o c}(x, \boldsymbol{y})$ of the solution of $(36)$ with the finite element approximation $u_{h}(x, \boldsymbol{y})$ of the solution of the nonlocal problem (32), using the same piecewise-linear finite element method for both. We measure the difference between the two approximate solutions using the metric

$$
\varepsilon_{\infty}=\max _{\boldsymbol{y} \in \Xi_{\text {test }}}\left\|\mid u_{h}-u_{h}^{l o c}\right\| \|_{h}
$$

The convergence of $u_{h}$ to $u_{h}^{l o c}$ as $\delta \rightarrow 0$ is illustrated in Figure 1 which also illustrates the linear convergence rate predicted by the theory.

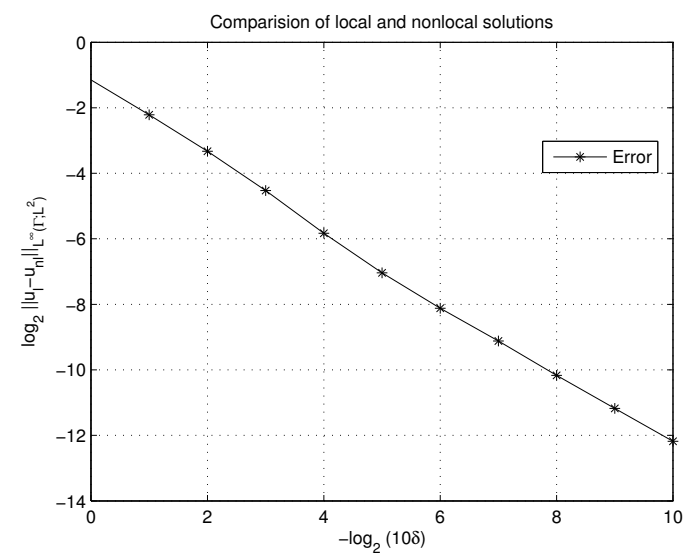

Figure 1: Differences between the solution of the nonlocal problem and the solution of the local problem for several values of the horizon $\delta$. 


\subsection{Comparison of the reduced-basis and full FE approaches for building surrogates}

It is well known that the online cost, i.e., the cost of evaluating $u_{r b 1}, u_{r b 2}$ and $u_{s g}$ are all very cheap, we focus on comparing the cost of constructing the three types of surrogates, i.e., the offline cost. There are several parameters appearing in the definition of the problem (32). In this section, we illustrate the effects that varying those parameters have on the relative performance of the three approximations. The constant $\widehat{a}$ is unimportant to our study; it just needs to be sufficiently large so that $a(x, \boldsymbol{y})$ is positive and bounded away from zero for all $x \in D \cup D_{I}, \boldsymbol{y} \in \Gamma$, and $N$. Thus, for all examples, we set $\widehat{a}=5$. We focus on illustrating the effects of varying the remaining parameters:

$\begin{array}{ll}h & \text { the spatial grid size } \\ N & \text { the number of random parameters } \\ \delta & \text { the horizon } \\ S & \text { the correlation length of the coefficient } \theta\left(x, x^{\prime} ; \boldsymbol{y}\right) \\ s & \text { the exponent in the kernel } \gamma\left(x, x^{\prime}\right)\end{array}$

For every case considered, we provide two plots, one each for the two error measures (34) and (35). Each plot contains three curves, one each for the three approximations RB1, RB2, and FEM. The errors are plotted vs. the total number of flops used to obtain the approximations. For the reduced-basis methods, the flop count grows as we increase the number of snapshots $K$; for the sparse-grid method, the flop count grows as we increase the sparse grid level $L$. Each of the five figures in this section corresponds to varying one of the five parameters in the list (37) while the other parameters are fixed. In reading the figures, one should keep in mind that within some of the figures, the axes values change from row to row so that visual examinations from a distance are not reliable.

We can draw the following conclusions from the figures.

1. The reduced-basis method RB2 outperforms the reduced-basis method RB1.

2. Except for low values of the sparse-grid level $L$, i.e., for a relatively small number of sparse-grid points, both reduced basis methods RB1 and RB2 outperform the direct finite element approach FEM.

3. As the cost of a full finite element solve increases, the advantages of the reduced basis methods RB1 and RB2 over the full finite element method increase.

4. As the number of finite element solves required increases, the advantages of the reduced basis methods RB1 and RB2 over the full finite element method FEM increase.

All methods require finite element solves. However, the reduced basis methods RB1 and RB2 only require such solves for the construction of the reduced basis. On the other hand, the direct finite element approach FEM uses the full finite element system (14) when constructing the sparse-grid interpolant and thus requires many more finite element solves compared to those required by the reduced basis methods.

In Figure 2, we vary the grid size $h$. Clearly, decreasing the spatial grid size $h$ increases the cost of the finite element solves. This is noteworthy because it indicates that the 
reduced-basis methods RB1 and RB2 will be even more advantageous, relative to the finite element approach FEM, for problems in two and three spatial dimensions.

In Figure 3, we vary the number of parameters $N$. For a given level $L$, increasing the number of random parameters $N$ results in an increase (in fact, a very rapid increase) in the number of sparse-grid points. Thus, the reduced basis methods RB1 and RB2, which solve reduced basis systems when constructing a sparse-grid interpolant, becomes increasingly more efficient as $N$ increases relative to the direct finite element approach FEM that uses full finite element solves for that purpose.

In Figure 4, we vary the horizon $\delta$. Increasing the horizon $\delta$ increases the extent of nonlocal interactions and thus increases the number of nonzero entries (as well as the bandwidth) of the finite element stiffness matrix. On the other hand, the reduced basis stiffness matrices are dense but are of much smaller dimension compared to the finite element stiffness matrix; furthermore, because they are dense, the cost of a reduced basis solve is unaffected by the value of $\delta$. As a result, because of nonlocality, increasing $\delta$ result in increasing finite element solve costs. From the figure, one sees that for the smallest value of $\delta=0.01$ and for the grid size $h=1 / 1024$, the reduced basis methods RB1 and RB2 are less efficient than the FEM approach unless the number of levels $L$ exceeds 10 . As the nonlocality increases, the cross-over point moves to the left; for $\delta=1$, RB2 is more efficient than FEM for $L>0$ and RB1 is more efficient for $L>2$. Of course, if we decrease $h$, the crossover points in all the plots will move to the left as illustrated in Figure 2. Again, the advantages of using reduced basis methods RB1 and RB2 relative to the finite element approach FEM will grow as the spatial dimension increases.

In Figure 5, we vary the correlation length $S$. The value of the correlation length $S$ seems to have a very small effect on the performances of the individual methods, and thus on the performance of the reduced basis methods RB1 and RB2 relative to the finite element approach FEM.

In Figure 6, we vary the kernel exponent $s$. The value of the kernel exponent $s$ seems to have a negligible effect on the performances of the individual methods, and thus on the performance of the reduced basis methods RB1 and RB2 relative to the finite element approach FEM. Note that we have included the case $s=-0.5$ in Figure 6 . We did so because for that value of $s$, the kernel $\gamma\left(x, x^{\prime}\right)$ is integrable, i.e., $\int_{\mathbb{R}} \gamma\left(x, x^{\prime}\right) d x^{\prime}<\infty$ for all $x \in \mathbb{R}$. Integrable kernels are of interest because in the nonlocal peridynamics model for solid mechanics, the kernels used in two and three dimensions are integrable. Thus, we do want to see if the integrability or non-integrability of the kernel makes much difference with regards to the advantage of using the reduced basis methods RB1 and RB2 relative to the finite element approach FEM; at least from Figure 6, it seems that it does not.

\subsection{Comparison of the error vs. the dimension of the approximation spaces}

We now compare, for different sparse-grid levels, errors for the three surrogates $u_{r b 1}$, $u_{r b 2}$ and $u_{s g}$ vs. the dimension of the surrogate spaces. For the reduced-basis surrogates, this dimensions is the number of reduced-basis vectors whereas for the surrogate built directly from full finite element solutions, that dimension is the number of sparse-grid 

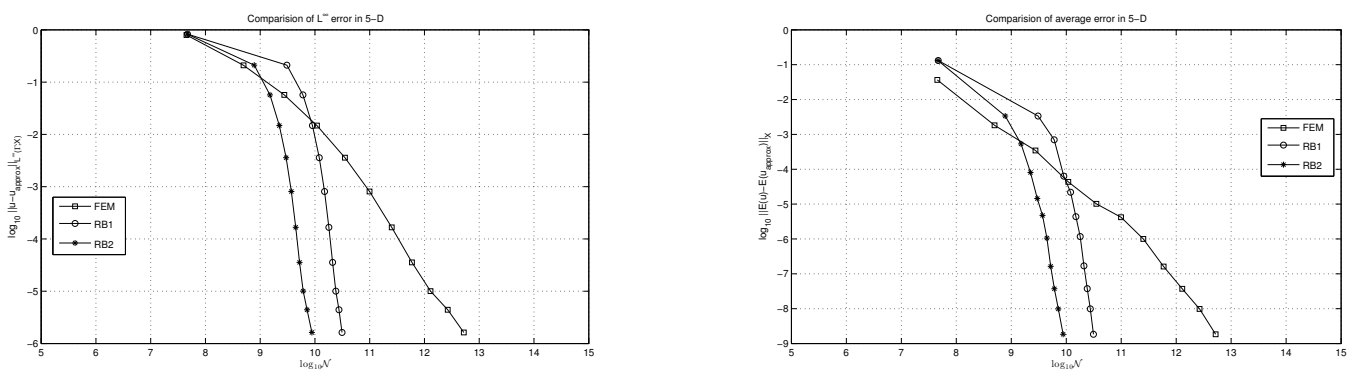

$h=1 / 512$
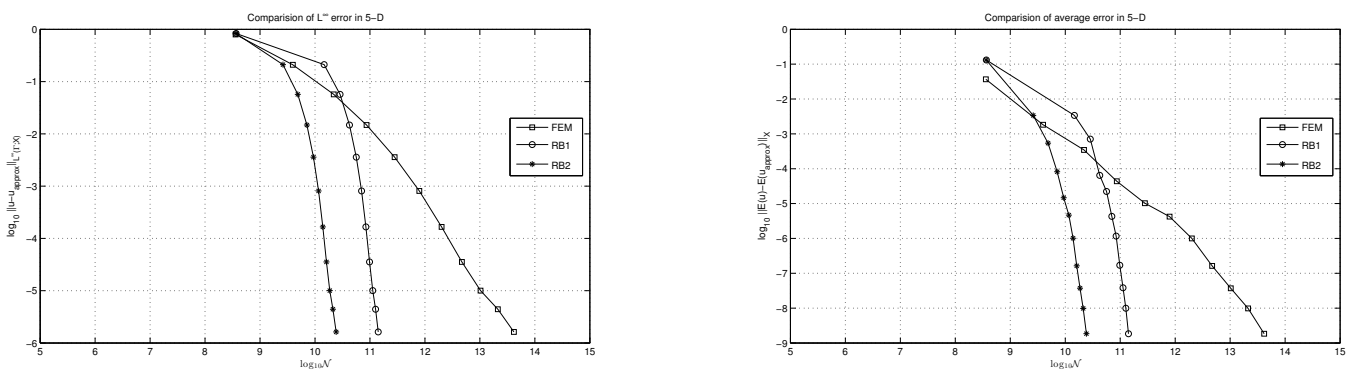

$$
h=1 / 1024
$$
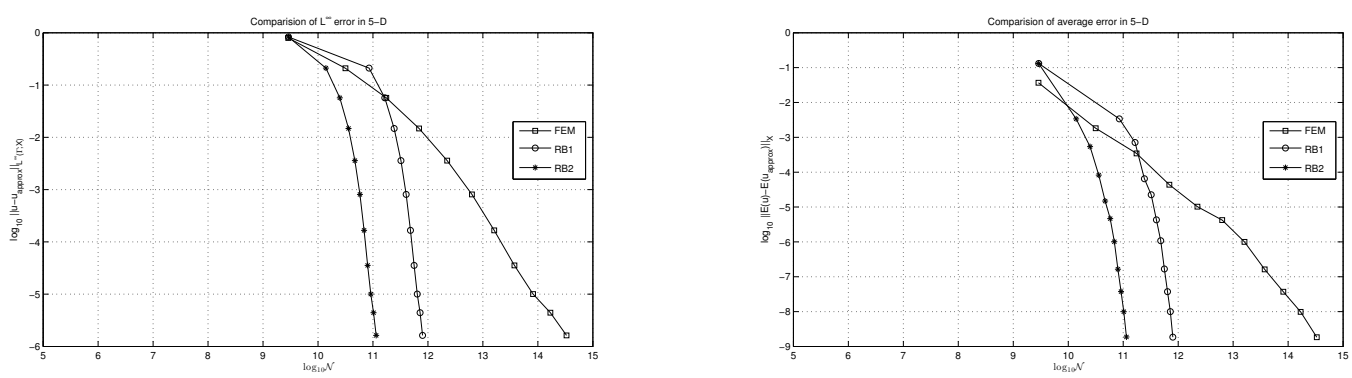

$$
h=1 / 2048
$$

Figure 2: Error measures $\varepsilon_{\infty}$ (left) and $\varepsilon_{\text {ave }}$ (right) for the three approximations vs. the number of flops $\mathcal{N}$ for three values of the grid size $h$. The other problem parameters are set to $N=5, \delta=1, S=1 / 2$, and $s=0$.

points in the sparse-grid level, i.e., the cardinality of the polynomial basis for the sparsegrid interpolant. Comparisons are made in Figure 7, in which, for the reduced-basis methods, $\mathcal{N}$ is now the number of reduced-basis vectors whereas for the full finite element surrogate, it is the number of sparse grid points. That figure gives results for several values of the horizon $\delta$. The marks on the curves for may be counted from left to right to determine the dimension of the bases. We again see the superior performance of the reduced-basis surrogates compared to the full finite element surrogate. 

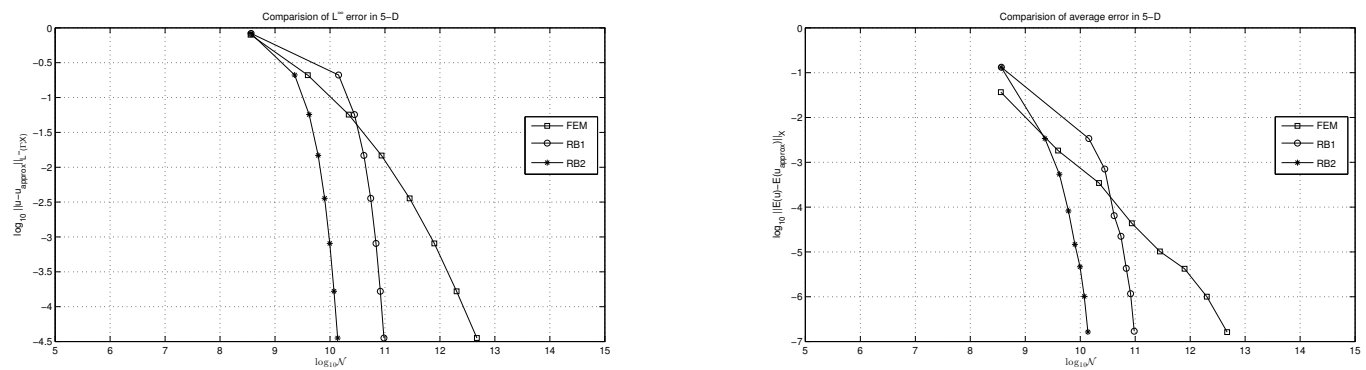

$N=5$
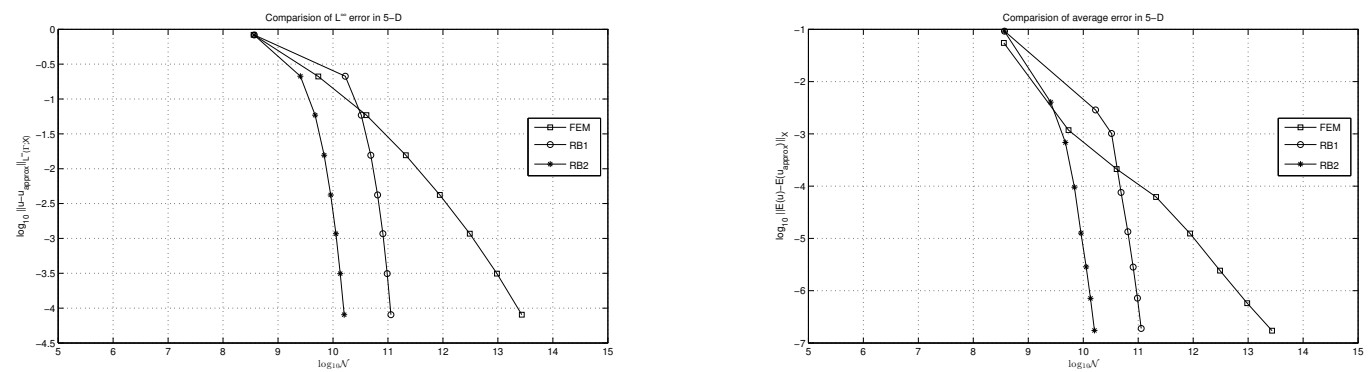

$N=7$
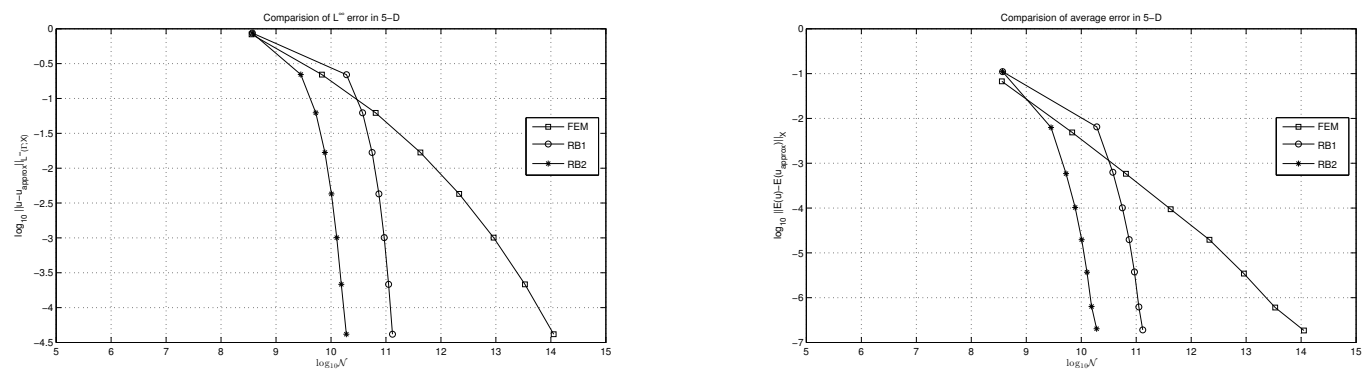

$N=9$

Figure 3: Error measures $\varepsilon_{\infty}$ (left) and $\varepsilon_{\text {ave }}$ (right) for the three approximations vs. the number of flops $\mathcal{N}$ for three values of number of parameters $N$. The other problem parameters are set to $h=1 / 1024$, $\delta=1, S=1 / 2$, and $s=0$.

\subsection{Comparison of the reduced-basis and the full FE approaches for approximating ex- pected values}

We now examine the performance of the reduced-basis surrogates relative to that of the sparse-grid surrogates for approximations of the expected values of the solution of the nonlocal model. We again consider the example of $\S 5.1$ for which the random parameters are uniformly distributed on $\Gamma=[-\sqrt{3}, \sqrt{3}]^{N}$. The "truth" value of the expected value is determined using a level 10 sparse-grid quadrature rule and the finite element approximate solutions $u_{h}(x, \boldsymbol{y})$. Denoting the set of level 10 sparse-grid points by $\Xi_{10} \subset \Gamma$ we then 

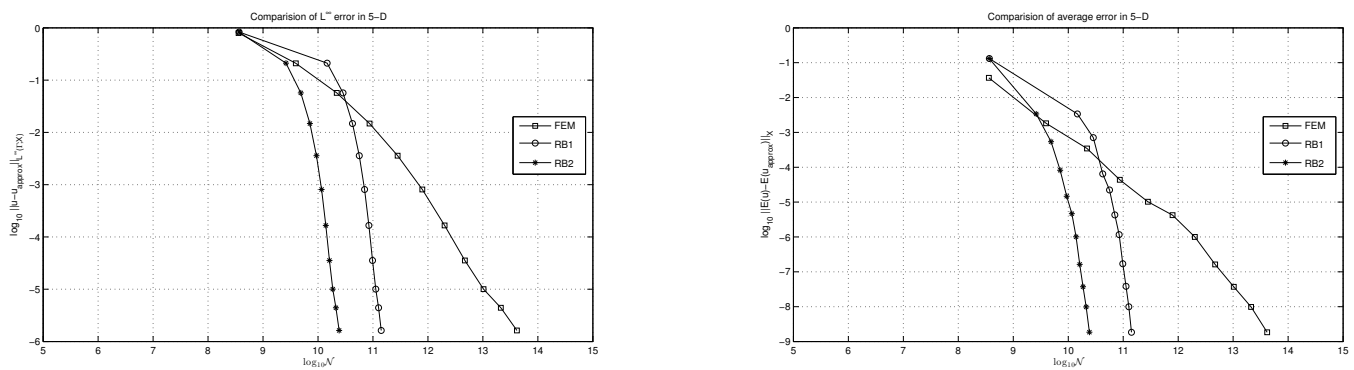

$\delta=0.5$
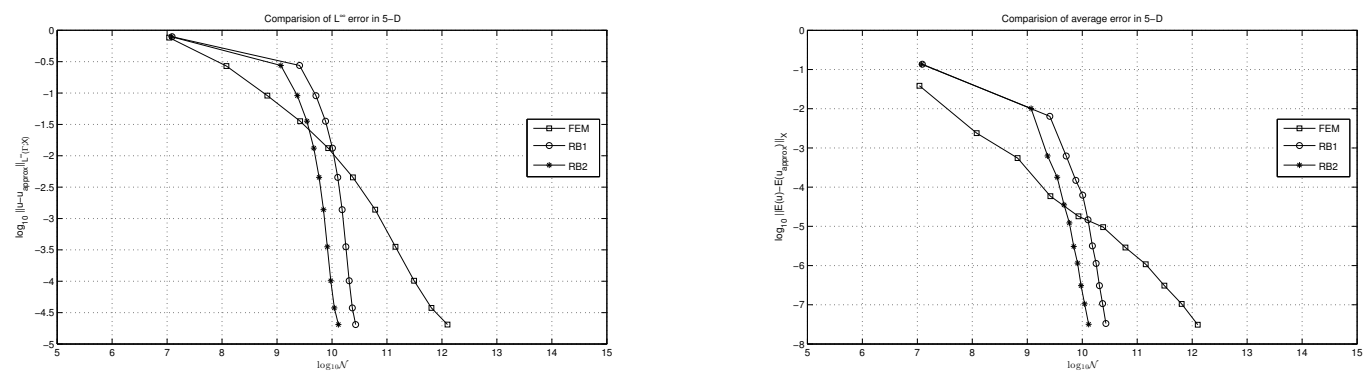

$$
\delta=0.1
$$
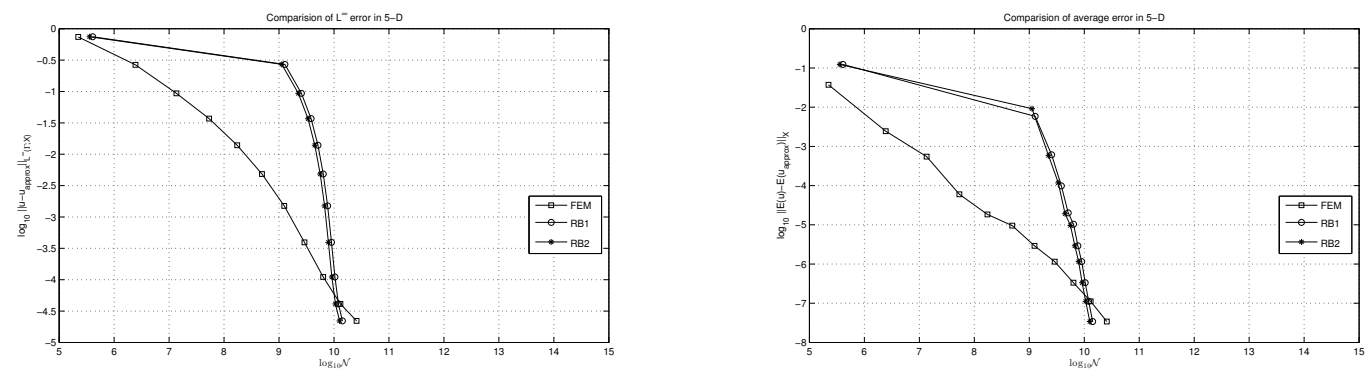

$\delta=0.01$

Figure 4: Error measures $\varepsilon_{\infty}$ (left) and $\varepsilon_{\text {ave }}$ (right) for the three approximations vs. the number of flops $\mathcal{N}$ for three values of the horizon $\delta$. The other problem parameters are set to $h=1 / 1024, N=5$, $S=1 / 2$, and $s=0$.

have that the expected value $\mathbb{E}[u](x)$ is replaced by the truth model

$$
\mathbb{E}_{10, h}[u](x)=\sum_{\boldsymbol{y} \in \Xi_{10}} w_{\boldsymbol{y} ; 10} u_{h}(x, \boldsymbol{y}) \approx \mathbb{E}[u](x)=\int_{\Gamma} u_{h}(x, \boldsymbol{y}) d \boldsymbol{y}
$$

where $w_{\boldsymbol{y} ; 10}$ is the quadrature weight corresponding to the sparse grid quadrature point $\boldsymbol{y} \in \Xi_{10}$. Then, for each of the three surrogates and for each sparse-grid level $\ell=0, \ldots, 8$, 

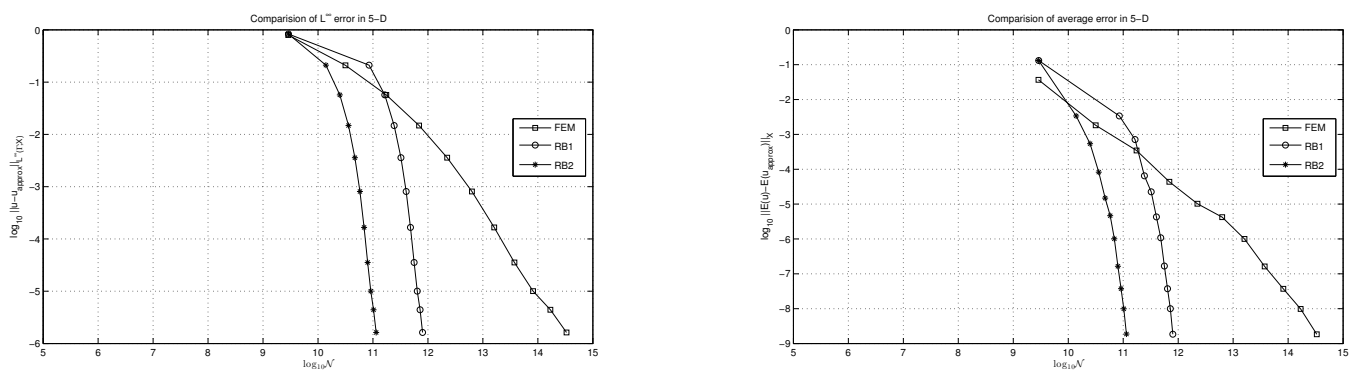

$S=1 / 2$
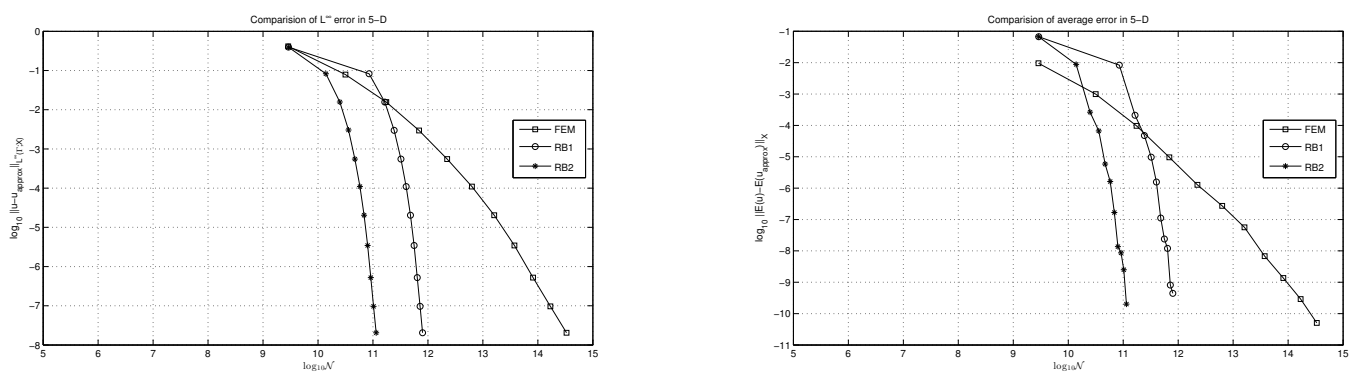

$$
S=1 / 8
$$

Figure 5: Error measures $\varepsilon_{\infty}$ (left) and $\varepsilon_{\text {ave }}$ (right) for the three approximations vs. the number of flops $\mathcal{N}$ for two values of the correlation length $S$. The other problem parameters are set to $h=1 / 2048$, $N=5, \delta=1$, and $s=0$.

the expected value is estimated by

$$
\mathbb{E}_{\ell, \text { surrogate }}[u](x):=\sum_{\boldsymbol{y} \in \Xi_{\ell}} w_{w_{\boldsymbol{y} ; \ell}} u_{\text {surrogate }}(x, \boldsymbol{y})
$$

where $\Xi_{\ell} \subset \Gamma$ denotes the set of level $\ell$ sparse-grid quadrature points. Then, we define the error in the expected value to be, for $\ell=0, \ldots, 8$,

$$
\varepsilon_{\ell, \text { surrogate }}=\left\|\mathbb{E}_{\ell, \text { surrogate }}(x)-\mathbb{E}_{10, h}(x)\right\|_{h} .
$$

In Figures 8 and 9, we provide plots of this error measures for each of the RB1, RB2, and FEM surrogates. The two figures examine the effects of changing the values of $\delta$ and $s$, respectively.

From the figures, one sees that for 40 basis functions, the two reduced basis surrogates have very similar errors as does the full finite element surrogate for all nine sparse-grid quadrature levels. For fewer basis functions, the reduced basis error plateaus at a sparsegrid quadrature level that increases with increasing number of reduced basis vectors. This is because with fewer number of basis vectors, the error made in the reduced basis approximation eventually dominates the error made by the sparse quadrature approximation. 

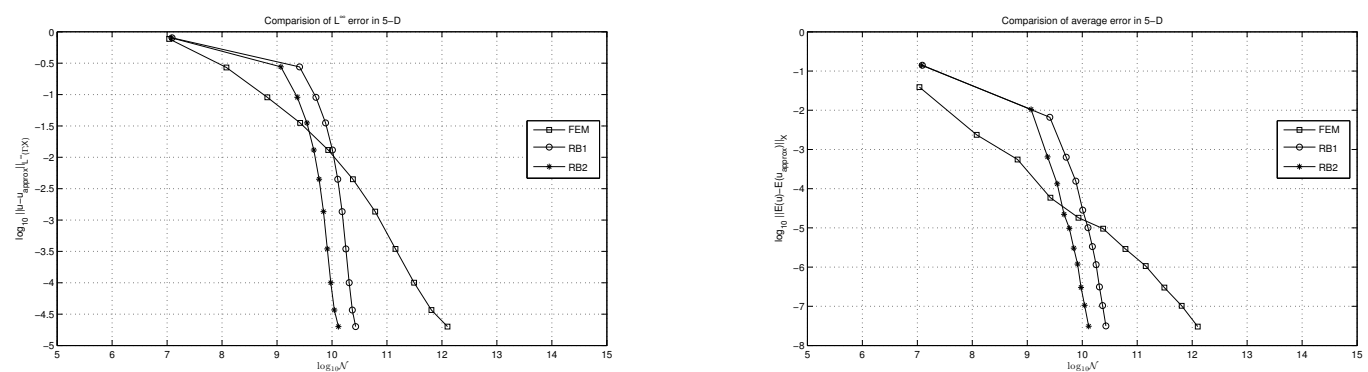

$s=-0.5$
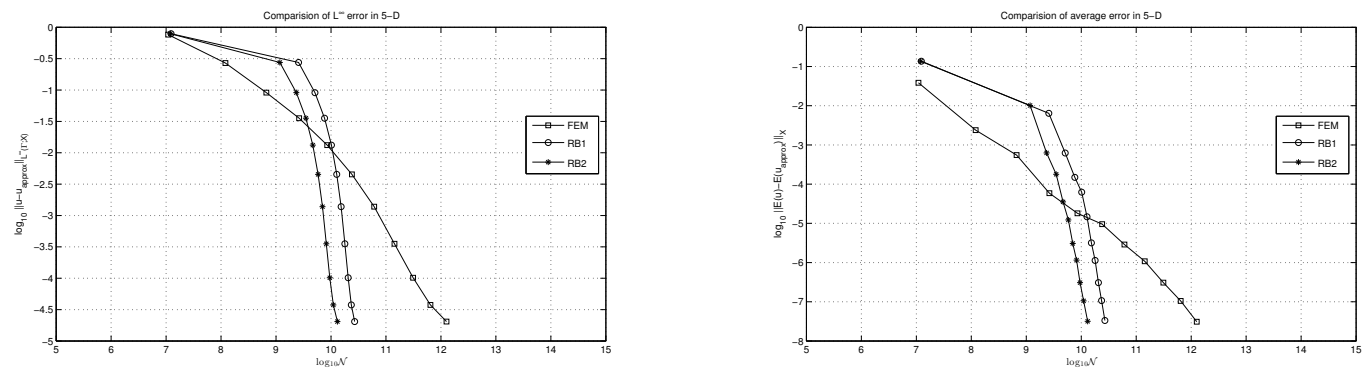

$s=0$
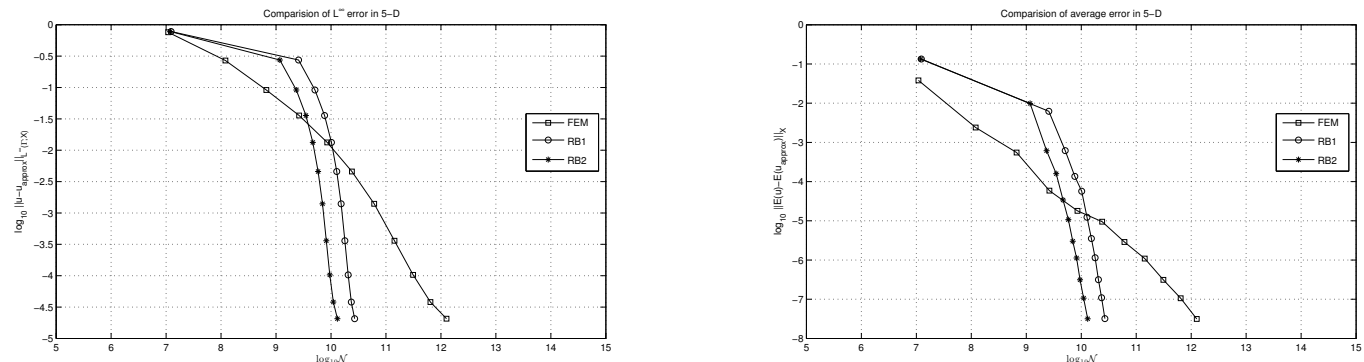

$s=0.25$

Figure 6: Error measures $\varepsilon_{\infty}$ (left) and $\varepsilon_{\text {ave }}$ (right) for the three approximations vs. the number of flops $\mathcal{N}$ for four values of the kernel exponent $s$. The other problem parameters are set to $h=1 / 1024, N=5$, $\delta=0.1$, and $S=1 / 2$.

Of course, this is not the case for the surrogate built from full finite element solutions; in fact we have that $\varepsilon_{10, \mathrm{fem}}=0$. On the other hand, once the reduced bases are determined, on can obtain the approximations or the expected values at much less cost for the reduced basis method that for the use of full finite element solutions. The overall error behavior seems insensitive to the values of the horizon $\delta$ and the exponent $s$. 

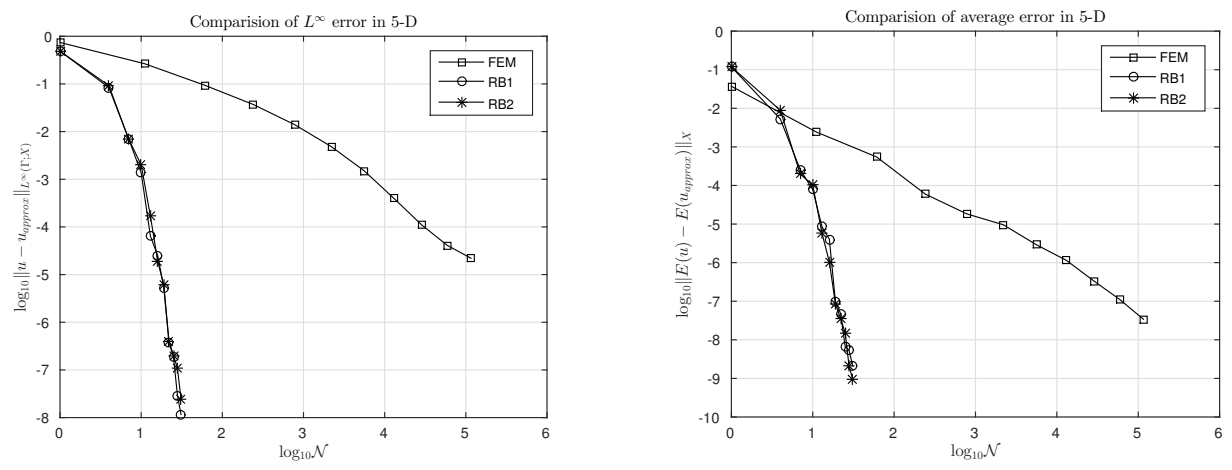

$\delta=0.01$
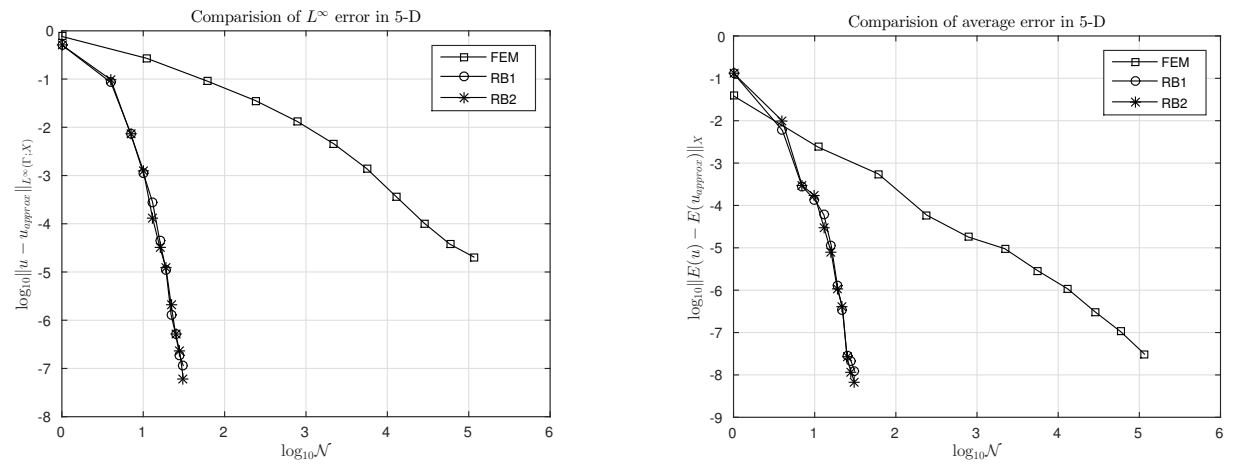

$\delta=0.1$
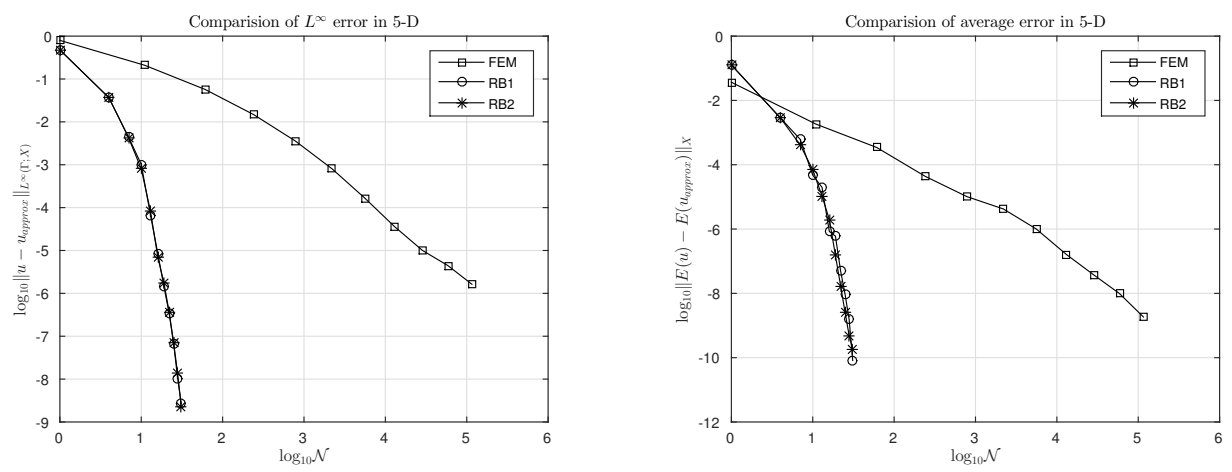

$\delta=1$

Figure 7: Error measures $\varepsilon_{\infty}$ (left) and $\varepsilon_{\text {ave }}$ (right) for the three approximations vs. the number of basis $\mathcal{N}$ for RB1,RB2 and the number of flops $\mathcal{N}$ for sparse-grid levels $L=0,1, \ldots, 10$ and for three values of the horizon $\delta$. The other problem parameters are set to $h=1 / 1024, N=5, S=1 / 2, s=0$. 

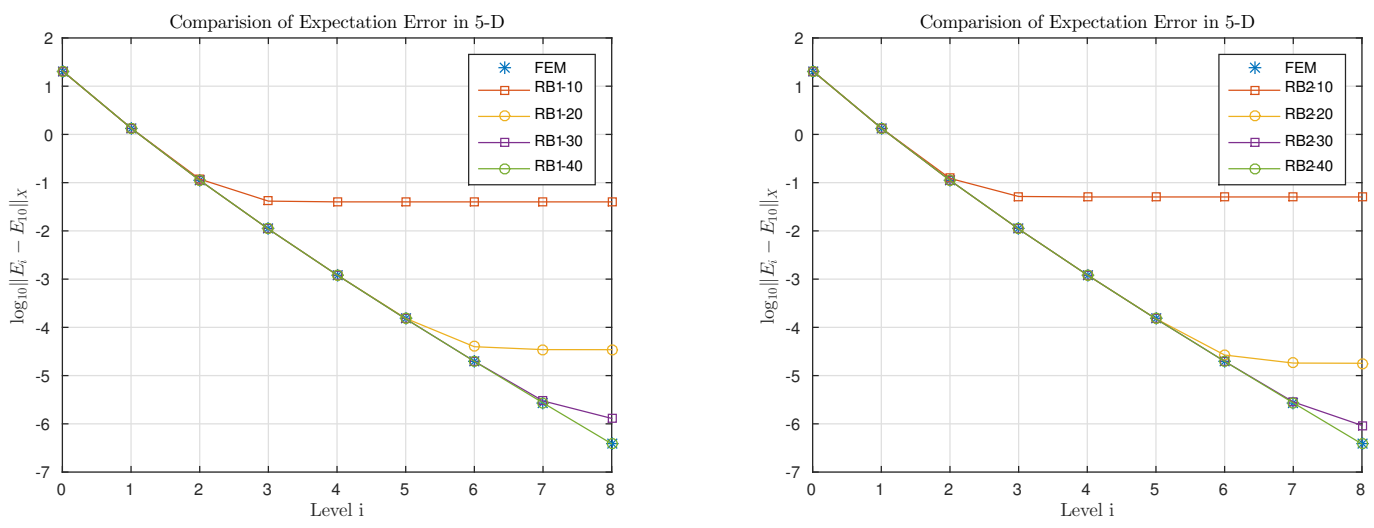

$\delta=0.01$
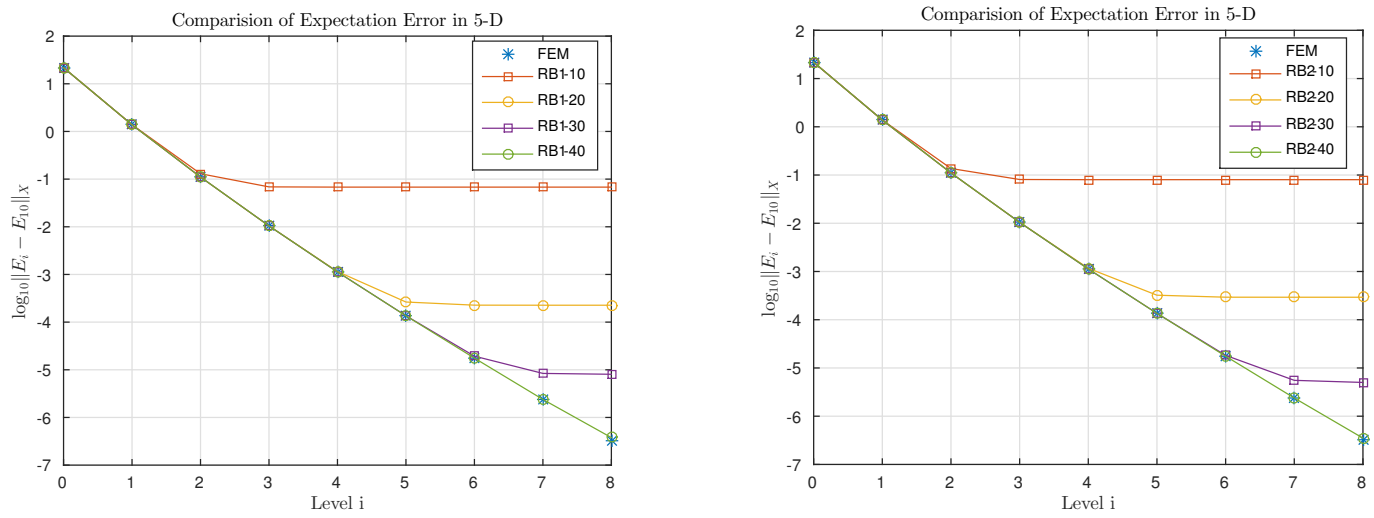

$\delta=0.1$
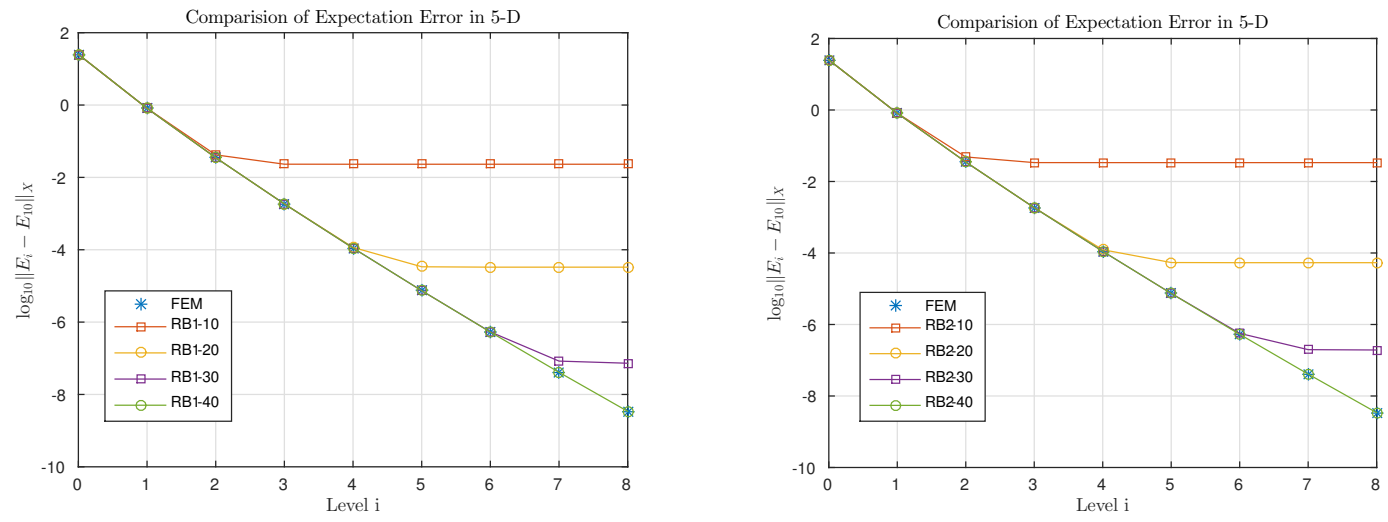

$$
\delta=1
$$

Figure 8: For four values of the horizon $\delta$ and for sparse-grid levels $\ell=0,1, \ldots, 8$, the error measures $\varepsilon_{\ell, \text { surrogate }}=\left\|\mathbb{E}_{\ell \text {,surrogate }}(x)-\mathbb{E}_{10, h}(x)\right\|_{h}$ with 10 to 40 basis functions for the RB1 (left) and RB2 (right) surrogates compared to the full finite element surrogate. The other problem parameters are set to $h=1 / 1024, N=5, S=1 / 2$, and $s=0$. The marks on the curves may be counted from left to right to determine the corresponding sparse-grid level $\ell$. 

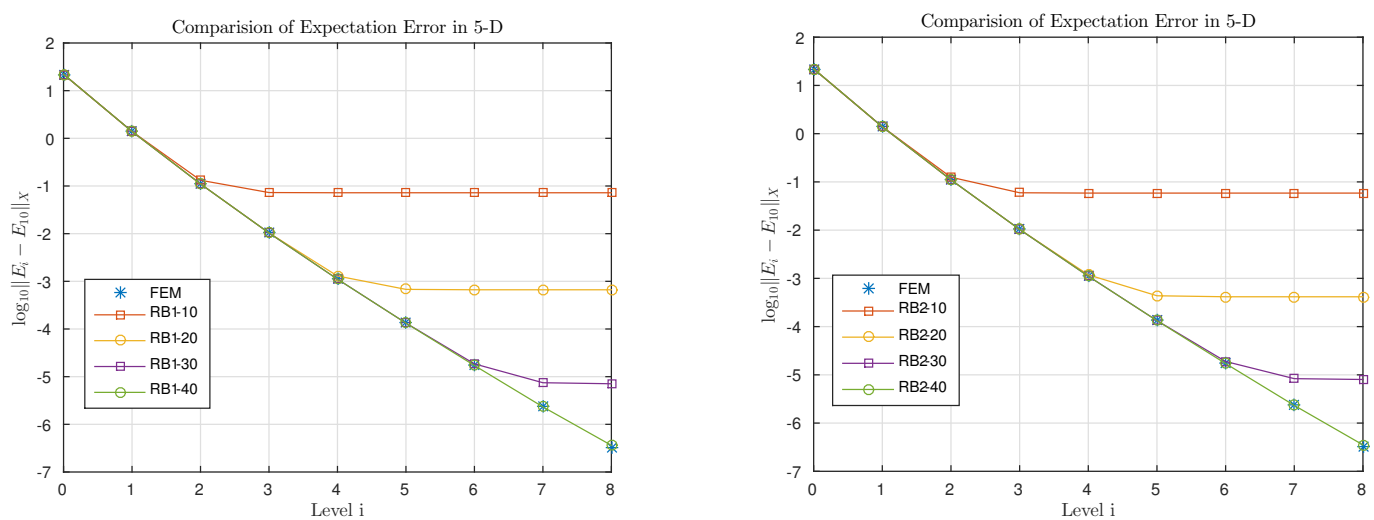

$s=-0.5$
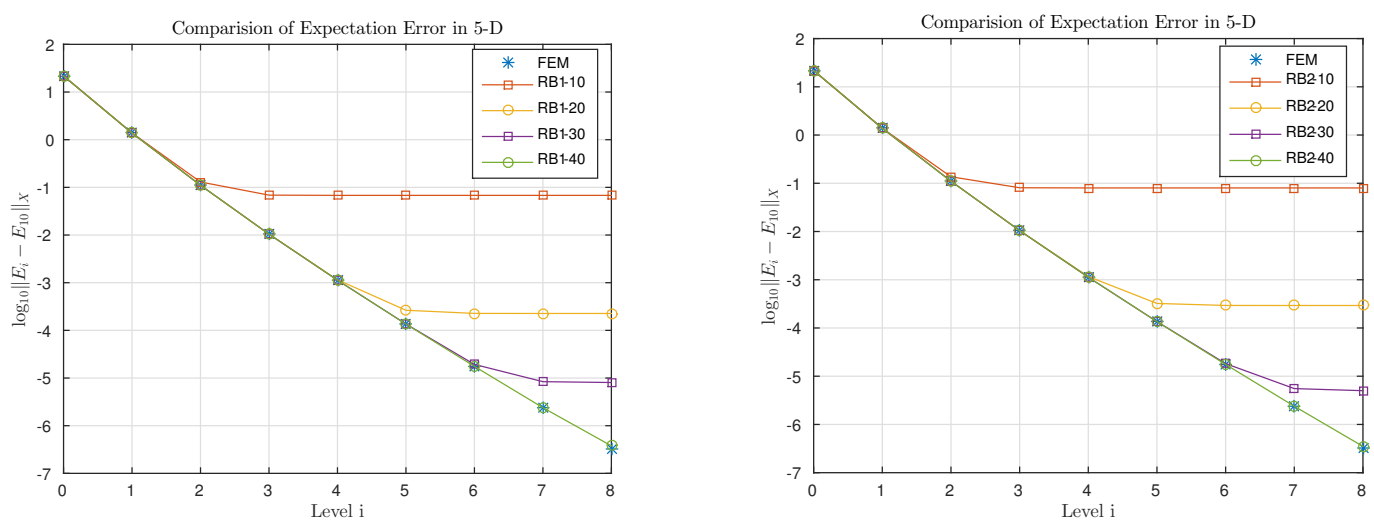

$s=0$
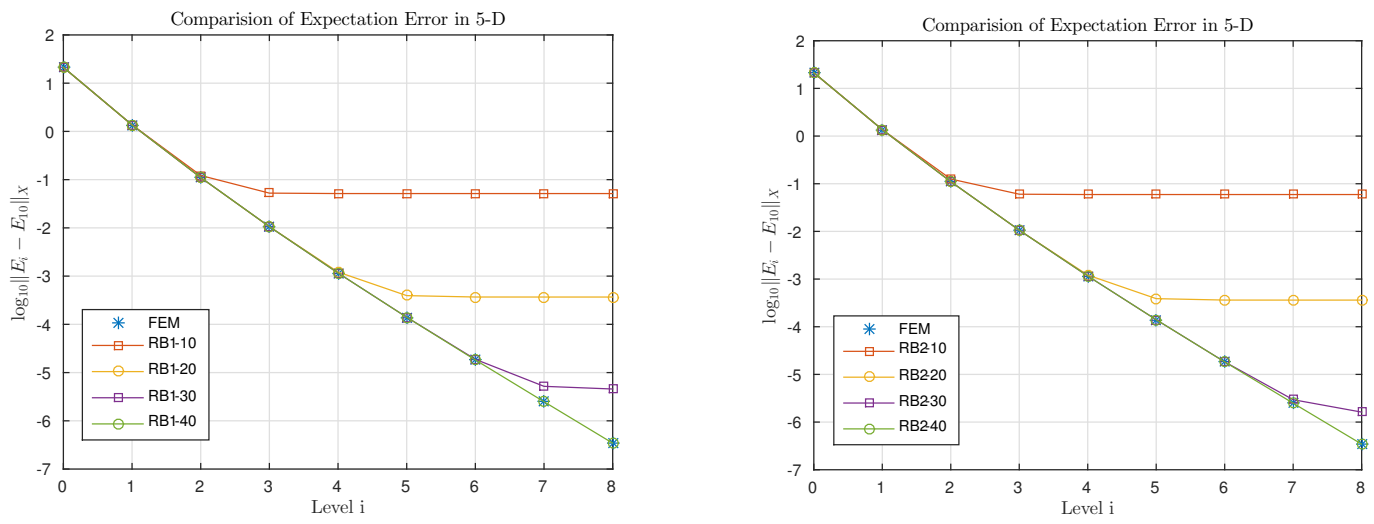

$s=0.5$

Figure 9: For four values of the exponent $s$ and for sparse-grid levels $\ell=0,1, \ldots, 8$, the error measures $\varepsilon_{\ell, \text { surrogate }}=\left\|\mathbb{E}_{\ell, \text { surrogate }}(x)-\mathbb{E}_{10, h}(x)\right\|_{h}$ with 10 to 40 basis functions for the RB1 (left) and RB2 (right) surrogates compared to the full finite element surrogate. The other problem parameters are set to $h=1 / 1024, N=5, S=1 / 2$, and $\delta=0.1$. The marks on the curves may be counted from left to right to determine the corresponding sparse-grid level $\ell$. 
[1] S. A. Silling, Reformulation of elasticity theory for discontinuities and long-range forces, J. Mech. Phys. Solids, 48(2000),pp.175-209.

[2] S. A. Silling, Linearized Theory of Peridynamic States, J. Elast (2010)99:85-111.

[3] A. Buades, B. Coll, J.M. Morel, Image denoising methods. A new nonlocal principle, SIAM Review(2010), Vol. 52, No. I, pp.113-147.

[4] G. Gilboa, S. Osher, Nonlocal linear image regularization and supervised segmentation, Multiscale Model. Simul., 6(2007), pp.595-630.

[5] G. Gilboa, S. Osher, Nonlocal operators with applications to image processing, Multiscale Model. Simul., 7(2008), pp.1005-1028.

[6] Y. Lou, X. Zhang, S. Osher, A. Bertozzi, Image recovery via nonlocal operators, J Sci Comput(2010) 42:185-197.

[7] R. Metzler, J. Klafter, The restaurant at the end of the random walk: recent developments in the description of anomalous transport by fractional dynamics, J. Phy. A: Math.Gen. 37(2004) R161-R20.8

[8] L. Rosasco, M. Belkin, E. De Vito, On learning with integral operators, J. Machine Learning Research 11(2010) pp.905-934.

[9] L. Lovász, B. Szegedy, Limits of dense graph sequences, J. Combinatorial Theory, Series B 96(2006) pp.933-957.

[10] Q. Du, M. Gunzburger, R. Lehoucq, K. Zhou, Analysis and approximation of nonlocal diffusion problems with volume constraints, 2012, SIAM Reivew, Vol.54, No.4, pp.667696.

[11] Q. Du, M. Gunzburger, R. Lehoucq, K. Zhou, A nonlocal vector calculus, nonlocal volume-constrained problems, and nonlocal balance laws, 2013, Math. Models Methods Appl. Sci. 23, 493.

[12] M. Stein, Large sample properties of simulations using Latin hypercube sampling, Technometrics, 29(1987), pp.143-151.

[13] W. Loh, On Latin hypercube sampling, Ann. Statist., 24(1996), pp.2058-2080.

[14] B. Fox, Strategies for Quasi-Monte Carlo, Kluwer, Dordrect, The Netherlands, 1999.

[15] H. Niederreiter, Random number generation and quasi-Monte Carlo methods, SIAM, Philadelphia, 1992.

[16] K. A. Cliffe, M. B. Giles, R. Scheichl, and A. L. Teckentrup, Multilevel Monte Carlo methods and applications to elliptic PDEs with random coefficients, Computing and Visualization in Science,14 (2011) 3-15. 
[17] I. Babuška, R. Tempone, G. E. Zouraris, Galerkin finite element approximations of stochastic elliptic partial differential equations, 2004, SIAM J. Numer. Anal., Vol.42, pp. 800-825.

[18] I. Babuška, R. Tempone, G. E. Zouraris, Solving elliptic boundary value problems with uncertian coefficients by the finite element method: The stochastic formulation, Comput. Methods Appl. Mech. Engrg., 194(2005), pp.1251-1294.

[19] O. L. Maritre, O. Knio, H. Najm, R. Ghanem, Uncertainty propagation using WienerHaar expansions, J. Comput. Phys., 197(2004), pp.28-57.

[20] H. G. Matthies, A. Keese, Galerkin methods for linear and nonlinear elliptic stochastic partial differential equations, Comput. Methods Appl. Mech. Engrg., 194(2005), pp.1295-1331.

[21] D. Xiu, G. Karniadakis, The Wiener-Askey polynomial chaos for stochastic differential equations, SIAM J. Sci. Comput., 24(2002), pp. 619-644.

[22] F. Nobile, R. Tempone and C. G. Webster, An anisotropic sparse grid stochastic collocation method for partial differential equations with random input data, 2008, SIAM Journal on Numerical Analysis Vol.46.5, 2411-2442.

[23] F. Nobile, R. Tempone and C. G. Webster, A sparse grid stochastic collocation method for partial differential equations with random input data, 2008, SIAM J. Numer. Anal, Vol.46, No.5, pp.2309-2345.

[24] X. Ma, N. Zabaras, An adaptive hierarchical sparse grid collocation algorithm for the solution of stochastic differential equations, J. Comput. Phys., 228(2009), pp.30843113.

[25] M. D'Elia, M. Gunzburger, Optimal distributed control of nonlocal steady diffusion problems. SIAM Journal on Control and Optimization 52.1 (2014): 243-273.

[26] X. Chen, M. Gunzburger, Continuous and discontinuous finite element methods for a peridynamics model of mechanics, Comput. Methods Appl. Mech. Engrg., 200 (2011), pp.1227-1250.

[27] G. Rozza, D. B. P. Huynh and A. T. Patera, Reduced Basis Approximation and a Posteriori Error Estimation for Affinely Parametrized Elliptic Coercive Partial Differential Equations, 2008, Arch Comput Methods Eng, 15: 229-275

[28] G. Rozza and K. Veroy, On the Stability of the Reduced Basis Method for Stokes Equations in Parametrized Domains. Computer Methods in Applied Mechanics and Engineering, 196(7): 1244-1260, 2007. 
[29] P. Chen, A. Quarteroni, and G. Rozza, Comparison Between Reduced Basis and Stochastic Collocation Methods for Elliptic Problems, 2014, Journal of Scientific Computing, Volume 59, Issue 1, pp. 187-216

[30] H. C. Elman and Q. Liao, Reduced Basis Collocation Methods for Partial Differential Equations with Random Coefficients, 2013, SIAM/ASA J. Uncertainty Quantification, 1(1), 192-217

[31] P. Chen, A. Quarteroni, and G. Rozza, A weighted reduced basis method for elliptic partial differential equations with random input data. SIAM Journal on Numerical Analysis 51.6 (2013): 3163-3185.

[32] I. Babuška, F. Nobile, R. Tempone, A stochastic collocation method for elliptic partial differential equations with random input data, 2007, SIAM J. Numer. Anal, Vol.45, No.3, pp.1005-1034.

[33] G. Zhang, M. Gunzburger, Error analysis of a stochastic collocation method for parabolic partial differential equations with random input data, 2012, SIAM J. Numer. Anal, Vol.50, No.4, pp.1922-1940.

[34] G. H. Golub, C. F. Van Loan, Matrix computations, (Johns Hopkins studies in mathematical sciences), (1996).

[35] S. P. Huang, S. T. Quek, and K. K. Phoon. Convergence study of the truncated K-L expansion for simulation of stochastic processes, International journal for numerical methods in engineering 52.9 (2001): 1029-1043.

[36] M. Meyer, H. G. Matthies, Efficient model reduction in non-linear dynamics using the Karhunen-Loeve expansion and dual-weighted-residual methods, (2003) Computational Mechanics, 31(1-2), 179-191.

[37] N. E. Gibbs, Jr, W. G. Poole, P. K. Stockmeyer, An algorithm for reducing the bandwidth and profile of a sparse matrix. SIAM Journal on Numerical Analysis, (1976) 13(2), 236-250.

[38] Q. Wang, X. W. Shi, An improved algorithm for matrix bandwidth and profile reduction in finite element analysis. Progress In Electromagnetics Research Letters, (2009) 9, 29-38.

[39] A. Gupta, F. G. Gustavson, M. Joshi, S. Toledo, The design, implementation, and evaluation of a symmetric banded linear solver for distributed-memory parallel computers. ACM Transactions on Mathematical Software (TOMS), (1998) 24(1), 74-101.

[40] M. Gunzburger, R. B. Lehoucq, A nonlocal vector calculus with application to nonlocal boundary value problems. Multiscale Modeling and Simulation, (2010) 8(5), 1581-1598. 
[41] Ronald DeVore, Guergana Petrova, and Przemyslaw Wojtaszczyk, Greedy algorithms for reduced basis in Banach spaces. Constructive Approximation, (2010) 8(5), 15811598.

[42] H. Tran, C. G. Webster and G. Zhang, Analysis of quasi-optimal polynomial approximations for parameterized PDEs with deterministic and stochastic coefficients, ORNL/TM-2014/468 Unlimited Release Printed September 2014.

\section{Appendix A. Smolyak sparse grids with Clenshaw-Curtis abscissas}

Recall that we have the product parameter domain $\Gamma=\prod_{n=1}^{N} \Gamma_{n}$. For each $n$, we select interpolation points $\left\{y_{n}^{m_{n}}\right\}_{m_{n}=1}^{M_{n}} \subset \Gamma_{n}$. Then, for $v\left(y_{n}\right) \in C^{0}\left(\Gamma^{n} ; V\right)$, the Lagrange interpolant corresponding to those points can be expressed in the form

$$
\mathcal{I}_{n}^{M_{n}}(v)\left(y_{n}\right)=\sum_{m_{n}=1}^{M_{n}} v\left(y_{n}^{m_{n}}\right) \ell_{n}^{m_{n}}\left(y_{n}\right) \quad \forall v \in C^{0}\left(\Gamma_{n} ; V\right) .
$$

$\mathcal{I}_{n}^{M_{n}}: C^{0}\left(\Gamma_{n} ; V\right) \rightarrow V_{n}\left(\Gamma_{n} ; V\right)$ is referred to as the Lagrange interpolation operator. In (A.1), $\ell_{n}^{m_{n}}\left(y_{n}\right) \in \mathcal{P}^{M_{n}-1}\left(\Gamma_{n}\right)$ for $n=1, \ldots, N$ are Lagrange polynomials of degree $M_{n}-1$ defined by $\ell_{n}^{m_{n}}\left(y_{n}\right)=\prod_{m=1, m \neq m_{n}}^{M_{n}} \frac{\left(y_{n}-y_{n}^{m}\right)}{\left(y_{n}^{m n}-y_{n}^{m}\right)}$ and

$$
V_{n}\left(\Gamma_{n} ; V\right)=\left\{v \in C^{0}\left(\Gamma_{n} ; V\right): v\left(y_{n}, \mathbf{x}\right)=\sum_{m_{l}=1}^{M_{l}} v_{k}(\mathbf{x}) \ell_{n}\left(y_{n}\right), v_{k}(\mathbf{x}) \in V\right\} .
$$

Using the collection of one-dimensional interpolation polynomials, on can define multivariate interpolants. For example, the full tensor product polynomial interpolant on $\Gamma \subset \mathbb{R}^{N}$ of the multivariate function $u(\boldsymbol{y})$ is defined by

$$
\begin{aligned}
u_{f t}(\boldsymbol{y}) & =\left(\mathcal{I}_{1}^{M_{1}} \otimes \cdots \otimes \mathcal{I}_{N}^{M_{N}}\right)[u(\boldsymbol{y})] \\
& =\sum_{m_{1}=1}^{M_{1}} \cdots \sum_{m_{N}=1}^{M_{N}} u\left(y_{1}^{m_{1}}, \cdots, y_{N}^{m_{N}}\right)\left(\ell_{1}^{m_{1}} \otimes \cdots \otimes \ell_{N}^{m_{N}}\right) .
\end{aligned}
$$

Another example is given by sparse grids polynomial interpolants; because these are a focus of this work, we give a brief description of how on class of sparse grid interpolants are constructed.

Sparse grids are defined as linear combinations of judiciously chosen tensor product points. As such, one first has to choose a family of one-dimensional interpolation points that is then used to build the multivariate tensor product interpolants. Here, for this purpose, we choose the Chebyschev family of one-dimensional points, or more precisely, a nested subset of that family. For each $n=1, \ldots, N$, we have the Clenshaw-Curtis (CC) family $\left\{y_{n}^{m_{n}}(l)\right\}_{m_{l}=1}^{M_{l}}$ of interpolation points, indexed by $l=1,2, \ldots$, given by

$$
y_{n}^{m_{n}}(l)=-\cos \left(\frac{\pi\left(m_{n}-1\right)}{M_{n}(l)-1}\right), \quad m_{n}=1, \ldots, M_{n}(l), \quad l=0, \ldots,
$$


where

$$
M_{n}(1)=1 \quad \text { and } \quad M_{n}(l)=2^{l-1}+1 \text { for } l>1 .
$$

Note that the number of interpolation points increase as the index $l$ increases, i.e., $M_{n}(l)>$ $M_{n}(l-1)$. Because of the way $M_{n}(l)$ is defined in (A.4), the interpolation points are nested, i.e., all interpolation points for indices $l^{\prime}=1, \ldots, l$ are included in the set of interpolation points for the index $l$. Nesting is important because it means that all the approximate solutions of the PDE obtained for constructing a level $l-1$ interpolant can be used to construct the level $l$ interpolant.

Sparse interpolation operators are based on linear combinations of tensor product operators (A.2). However, only certain products with relatively fewer points are used. We denote by $L \in \mathbb{N}_{+}$the desired level of the sparse grid interpolant. There are good reasons with regards to efficiency for choosing a different level for each $n$, but for the sake of simplicity, we assume that the level is the same for all $n$. We set the multi-index $\boldsymbol{l}=\left(l_{1}, \ldots, l_{N}\right)$, let $|\boldsymbol{l}|=\sum_{n=1}^{N} l_{n}$, and define the two sets of multi-indices

$$
\left\{\begin{array}{l}
\widetilde{\boldsymbol{l}}(L, N)=\left\{\boldsymbol{l} \in \mathbb{N}_{+}^{N}: \sum_{n=1}^{N}\left(l_{n}-1\right) \leq L\right\} \\
\widehat{\boldsymbol{l}}(L, N)=\left\{\boldsymbol{l} \in \mathbb{N}_{+}^{N}: L-N+1 \leq \sum_{n=1}^{N}\left(l_{n}-1\right) \leq L\right\} .
\end{array}\right.
$$

Then, the CC distinct sparse grid points are given by

$$
\bigcup_{\boldsymbol{l} \in \widetilde{\boldsymbol{l}}(L, N)} \bigotimes_{n=1}^{N}\left\{y_{n}^{m_{n}}(l)\right\}_{m_{n}=1}^{M_{n}\left(l_{n}\right)}
$$

and the corresponding sparse grid interpolation operator is given by

$$
u_{s g}(\boldsymbol{y})=\mathcal{I}_{L, N}[u(\boldsymbol{y})]=\sum_{\boldsymbol{l} \in \widehat{\boldsymbol{l}}(L, N)}(-1)^{L+N-|\boldsymbol{l}|}\left(\begin{array}{c}
N-1 \\
L+N-|\boldsymbol{l}|
\end{array}\right)\left(\mathcal{I}^{M_{1}\left(l_{1}\right)} \otimes \cdots \otimes \mathcal{I}^{M_{N}\left(l_{N}\right)}\right)[u(\boldsymbol{y})] .
$$

Only values of a function $u(\boldsymbol{y})$ at the sparse grid points (A.5) are needed to construct the sparse grid interpolant $\mathcal{I}_{L, N}[u]$.

\section{Appendix A.1. Convergence analysis}

In this section, we quote an error estimate for sparge grid interpolation. For any $\boldsymbol{y} \in \Gamma$, let $u_{h}(\cdot, \boldsymbol{y})$ denote the finite element approximation of the solution $u(\cdot, \boldsymbol{y})$ of $(5)$ and, for any $\mathbf{x} \in D$, let $\mathcal{I}_{L, N}\left[u_{h}(\cdot, \boldsymbol{y})\right]$ denote the level $L$ sparse grid interpolant of $u_{h}(\cdot, \boldsymbol{y})$. The error between $u$ and its sparse grid interpolant $\mathcal{I}_{L, N}\left[u_{h}\right]$ can be estimating by separately estimating the error $u-u_{h}$ due to spatial approximation and the error $u_{h}-\mathcal{I}_{L, N}\left[u_{h}\right]$ due to interpolation in parameter space. An estimate for the former is given in (7) whereas the latter is the subject of interest here. For a function $u(\cdot, \boldsymbol{y})$ that is holomorphic in an open neighborhood of $\mathbb{C}^{N}$, as described in [42, Theorem 1], then we have the following result: 
Theorem 1. For the nested Clenshaw-Curtis Smolyak sparse grid, we have that

$$
\left\|u_{h}-\mathcal{I}_{L, N}\left[u_{h}\right]\right\|_{L^{\infty}\left(\Gamma ; \mathcal{V}_{c}\right)} \leq C M e^{-M^{1 / N}}
$$

where $M$ denotes the number of collocation (i.e., interpolation) points and the value of the constant $C>0$ is related to the Lebesgue constant and is independent of the spatial mesh size $h$.

Proof. The proof follows that of the proof of [23, Theorem 3.10] and [42]. 